\title{
Variable Importance Plots-An Introduction to the vip Package
}

by Brandon M. Greenwell, Bradley C. Boehmke

\begin{abstract}
In the era of "big data", it is becoming more of a challenge to not only build state-of-the-art predictive models, but also gain an understanding of what's really going on in the data. For example, it is often of interest to know which, if any, of the predictors in a fitted model are relatively influential on the predicted outcome. Some modern algorithms-like random forests (RFs) and gradient boosted decision trees (GBMs) - have a natural way of quantifying the importance or relative influence of each feature. Other algorithms-like naive Bayes classifiers and support vector machines-are not capable of doing so and model-agnostic approaches are generally used to measure each predictor's importance. Enter vip, an R package for constructing variable importance scores/plots for many types of supervised learning algorithms using model-specific and novel model-agnostic approaches. We'll also discuss a novel way to display both feature importance and feature effects together using sparklines, a very small line chart conveying the general shape or variation in some feature that can be directly embedded in text or tables.
\end{abstract}

\section{Introduction}

Too often machine learning (ML) models are summarized using a single metric (e.g., cross-validated accuracy) and then put into production. Although we often care about the predictions from these models, it is becoming routine (and good practice) to also better understand the predictions! Understanding how an ML model makes its predictions helps build trust in the model and is the fundamental idea of the emerging field of interpretable machine learning (IML). ${ }^{1}$ For an in-depth discussion on IML, see Molnar (2019b). In this paper, we focus on global methods for quantifying the importance ${ }^{2}$ of features in an ML model; that is, methods that help us understand the global contribution each feature has to a model's predictions. Computing variable importance (VI) and communicating them through variable importance plots (VIPs) is a fundamental component of IML and is the main topic of this paper.

While many of the procedures discussed in this paper apply to any model that makes predictions, it should be noted that these methods heavily depend on the accuracy and importance of the fitted model; hence, unimportant features may appear relatively important (albeit not predictive) in comparison to the other included features. For this reason, we stress the usefulness of understanding the scale on which VI scores are calculated and take that into account when assessing the importance of each feature and communicating the results to others. Also, we should point out that this work focuses mostly on post-hoc interpretability where a trained model is given and the goal is to understand what features are driving the model's predictions. Consequently, our work focuses on functional understanding of the model in contrast to the lower-level mechanistic understanding (Montavon et al., 2018). That is, we seek to explain the relationship between the model's prediction behavior and features without explaining the full internal representation of the model. ${ }^{3}$

VI scores and VIPs can be constructed for general ML models using a number of available packages. The iml package (Molnar, 2019a) provides the FeatureImp() function which computes feature importance for general prediction models using the permutation approach (discussed later). It is written in R6 (Chang, 2019) and allows the user to specify a generic loss function or select one from a pre-defined list (e.g., loss = "mse" for mean squared error). It also allows the user to specify whether importance is measured as the difference or as the ratio of the original model error and the model error after permutation. The user can also specify the number of repetitions used when permuting each feature to help stabilize the variability in the procedure. The iml : :FeatureImp() function can also be run in parallel using any parallel backend supported by the foreach package (Revolution Analytics and Weston).

The ingredients package (Biecek et al., 2019a) also provides permutation-based VI scores through the feature_importance() function. (Note that this function recently replaced the now deprecated DALEX function variable_importance() (Biecek, 2019).) Similar to iml: :FeatureImp(), this function allows the user to specify a loss function and how the importance scores are computed (e.g., using the

\footnotetext{
${ }^{1}$ Although "interpretability" is difficult to formally define in the context of ML, we follow Doshi-Velez and Kim (2017) and describe "interpretable" as the "... ability to explain or to present in understandable terms to a human."

${ }^{2}$ In this context "importance" can be defined in a number of different ways. In general, we can describe it as the extent to which a feature has a "meaningful" impact on the predicted outcome. A more formal definition and treatment can be found in van der Laan (2006).

${ }^{3}$ We refer the reader to Poulin et al. (2006), Caruana et al. (2015), Bibal and Frénay (2016), and Bau et al. (2017), for discussions around model structure interpretation.
} 
difference or ratio). It also provides an option to sample the training data before shuffling the data to compute importance (the default is to use n_sample = 1000), which can help speed up computation.

The mmpf package (Jones, 2018) also provides permutation-based VI scores via the mmpf: : permutationImportance() function. Similar to the iml and ingredients implementation, this function is flexible enough to be applied to any class of ML models in R.

The varImp package (Probst, 2019) extends the permutation-based method for RFs in package party (Hothorn et al., 2019) to arbitrary measures from the measures package (Probst, 2018). Additionally, the functions in varImp include the option of using the conditional approach described in Strobl et al. (2008) which is more reliable in the presence of correlated features. A number of other RF-specific VI packages exist on CRAN, including, but not limited to, vita (Celik, 2015), rfVarImpOOB (Loecher, 2019), randomForestExplainer (Paluszynska et al., 2019), and tree.interpreter (Sun, 2019). .

The caret package (Kuhn, 2020) includes a general var Imp () function for computing model-specific and filter-based VI scores. Filter-based approaches, which are described in Kuhn and Johnson (2013), do not make use of the fitted model to measure VI. They also do not take into account the other predictors in the model. For regression problems, a popular filter-based approach to measuring the VI of a numeric predictor $x$ is to first fit a flexible nonparametric model between $x$ and the target $Y$; for example, the locally-weighted polynomial regression (LOWESS) method developed by Cleveland (1979). From this fit, a pseudo- $R^{2}$ measure can be obtained from the resulting residuals and used as a measure of VI. For categorical predictors, a different method based on standard statistical tests (e.g., $t$-tests and ANOVAs) can be employed; see Kuhn and Johnson (2013) for details. For classification problems, an area under the ROC curve (AUC) statistic can be used to quantify predictor importance. The AUC statistic is computed by using the predictor $x$ as input to the ROC curve. If $x$ can reasonably separate the classes of $Y$, that is a clear indicator that $x$ is an important predictor (in terms of class separation) and this is captured in the corresponding AUC statistic. For problems with more than two classes, extensions of the ROC curve or a one-vs-all approach can be used.

If you use the mlr interface for fitting ML models (Bischl et al., 2020), then you can use the getFeatureImportance () function to extract model-specific VI scores from various tree-based models (e.g., RFs and GBMs). Unlike caret, the model needs to be fit via the mlr interface; for instance, you cannot use getFeatureImportance() on a ranger (Wright et al., 2020) model unless it was fit using mlr.

While the iml and DALEX packages provide model-agnostic approaches to computing VI, caret, and to some extent, mlr, provide model-specific approaches (e.g., using the absolute value of the $t$-statistic for linear models) as well as less accurate filter-based approaches. Furthermore, each package has a completely different interface (e.g., iml is written in R6). The vip package (Greenwell et al., 2019) strives to provide a consistent interface to both model-specific and model-agnostic approaches to feature importance that is simple to use. The three most important functions exported by vip are described below:

- vi() computes VI scores using model-specific or model-agnostic approaches (the results are always returned as a tibble (Müller and Wickham, 2019));

- $\operatorname{vip}()$ constructs VIPs using model-specific or model-agnostic approaches with ggplot2-style graphics (Wickham et al., 2019);

- add_sparklines() adds a novel sparkline representation of feature effects (e.g., partial dependence plots) to any VI table produced by vi().

There's also a function called vint () (for variable interactions) but it is experimental and will not be discussed here; the interested reader is pointed to Greenwell et al. (2018). Note that vi() is actually a wrapper around four workhorse functions, vi_model(), vi_firm(), vi_permute(), and vi_shap(), that compute various types of VI scores. The first computes model-specific VI scores, while the latter three produce model-agnostic ones. The workhorse function that actually gets called is controlled by the method argument in $\mathrm{vi}()$; the default is method = "model" which corresponds to model-specific VI (see ?vip: : vi for details and links to further documentation).

\section{Constructing VIPs in $\mathbf{R}$}

We'll illustrate major concepts using the Friedman 1 benchmark problem described in Friedman (1991) and Breiman (1996):

$$
Y_{i}=10 \sin \left(\pi X_{1 i} X_{2 i}\right)+20\left(X_{3 i}-0.5\right)^{2}+10 X_{4 i}+5 X_{5 i}+\epsilon_{i}, \quad i=1,2, \ldots, n,
$$

\footnotetext{
${ }^{4}$ These packages were discovered using pkgsearch's ps() function (Csárdi and Salmon, 2019) with the key phrases "variable importance" and "feature importance".
} 
where $\epsilon_{i} \stackrel{i i d}{\sim} N\left(0, \sigma^{2}\right)$. Data from this model can be generated using the vip: : gen_friedman(). By default, the features consist of 10 independent variables uniformly distributed on the interval $[0,1]$; however, only 5 out of these 10 are actually used in the true model. The code chunk below simulates 500 observations from the model in Equation (1) with $\sigma=1$; see ?vip: :gen_friedman for details.

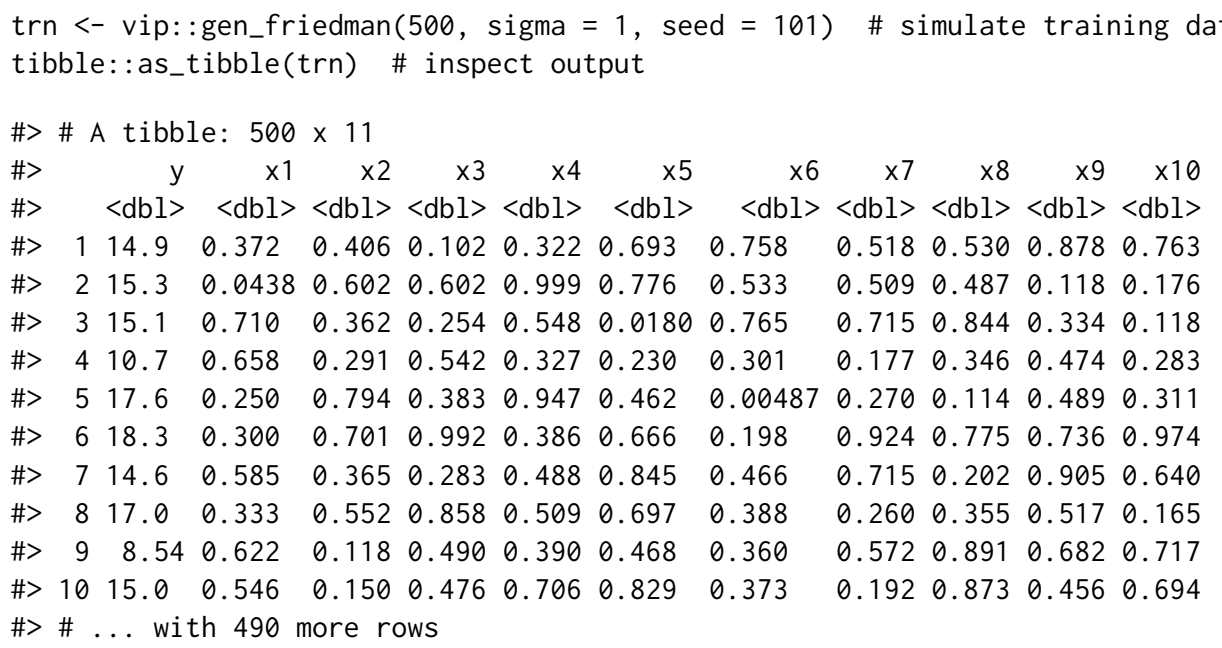

From Equation (1), it should be clear that features $X_{1}-X_{5}$ are the most important! (The others don't influence $Y$ at all.) Also, based on the form of the model, we'd expect $X_{4}$ to be the most important feature, probably followed by $X_{1}$ and $X_{2}$ (both comparably important), with $X_{5}$ probably being less important. The influence of $X_{3}$ is harder to determine due to its quadratic nature, but it seems likely that this nonlinearity will suppress the variable's influence over its observed range (i.e., $0-1$ ).

\section{Model-specific VI}

Some machine learning algorithms have their own way of quantifying the importance of each feature, which we refer to as model-specific VI. We describe some of these in the subsections that follow. One particular issue with model-specific VI scores is that they are not necessarily comparable across different types of models. For example, directly comparing the impurity-based VI scores from treebased models to the the absolute value of the $t$-statistic in linear models.

\section{Decision trees and tree ensembles}

Decision trees probably offer the most natural model-specific approach to quantifying the importance of each feature. In a binary decision tree, at each node $t$, a single predictor is used to partition the data into two homogeneous groups. The chosen predictor is the one that maximizes some measure of improvement $i^{t}$. The relative importance of predictor $X$ is the sum of the squared improvements over all internal nodes of the tree for which $X$ was chosen as the partitioning variable; see Breiman et al. (1984) for details. This idea also extends to ensembles of decision trees, such as RFs and GBMs. In ensembles, the improvement score for each predictor is averaged across all the trees in the ensemble. Fortunately, due to the stabilizing effect of averaging, the improvement-based VI metric is often more reliable in large ensembles; see Hastie et al. (2009, p. 368).

RFs offer an additional method for computing VI scores. The idea is to use the leftover out-of-bag (OOB) data to construct validation-set errors for each tree. Then, each predictor is randomly shuffled in the OOB data and the error is computed again. The idea is that if variable $X$ is important, then the validation error will go up when $X$ is perturbed in the OOB data. The difference in the two errors is recorded for the OOB data then averaged across all trees in the forest. Note that both methods for constructing VI scores can be unreliable in certain situations; for example, when the predictor variables vary in their scale of measurement or their number of categories (Strobl et al., 2007), or when the predictors are highly correlated (Strobl et al., 2008). The varImp package discussed earlier provides methods to address these concerns for random forests in package party, with similar functionality also built into the partykit package (Hothorn and Zeileis, 2019). The vip package also supports the conditional importance described in (Strobl et al., 2008) for both party- and partykit-based RFs; see ?vip: : vi_model for details. Later on, we'll discuss a more general permutation method that can be applied to any supervised learning model. 
To illustrate, we fit a CART-like regression tree, RF, and GBM to the simulated training data. (Note: there are a number of different packages available for fitting these types of models, we just picked popular implementations for illustration.)

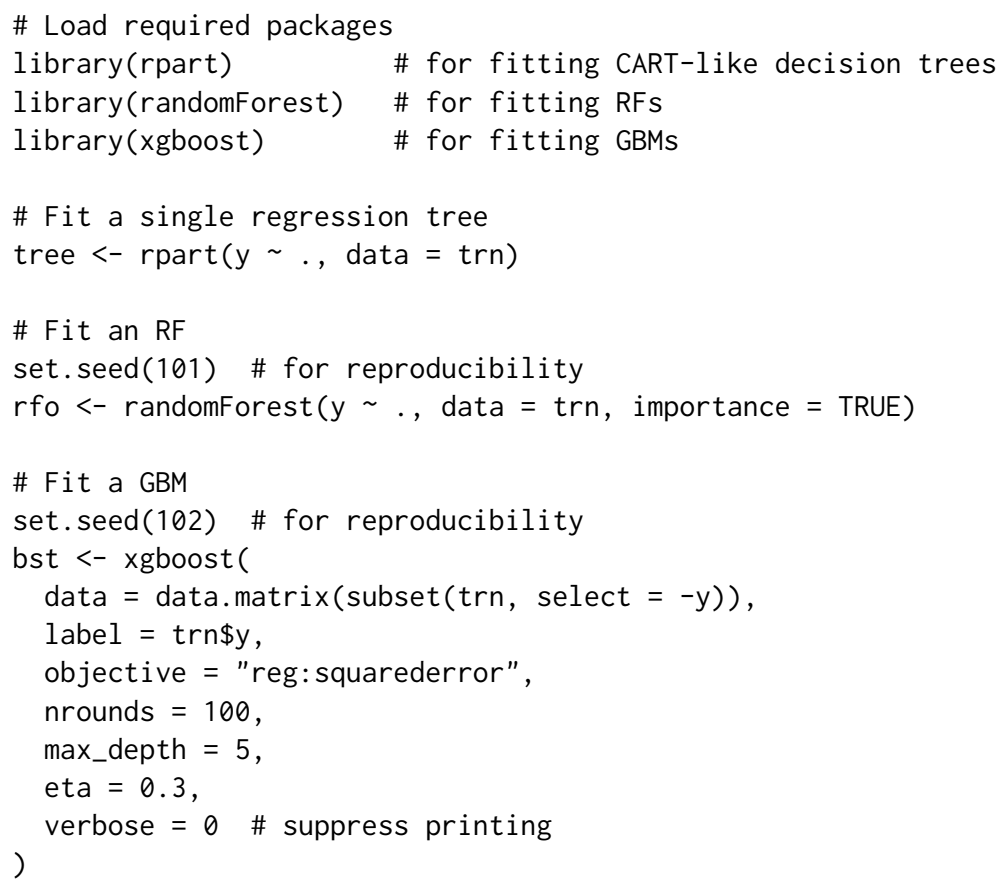

Each of the above packages include the ability to compute VI scores for all the features in the model; however, the implementation is rather package-specific, as shown in the code chunk below. The results are displayed in Figure 1 (the code to reproduce these plots has been omitted but can be made available upon request).

\# Extract VI scores from each model $v i \_$tree <- tree\$variable.importance vi_rfo <- rfo\$variable.importance \# or use 'randomForest::importance( $r f o)^{-}$ vi_bst $<-x g b$.importance $($ model $=$ bst $)$
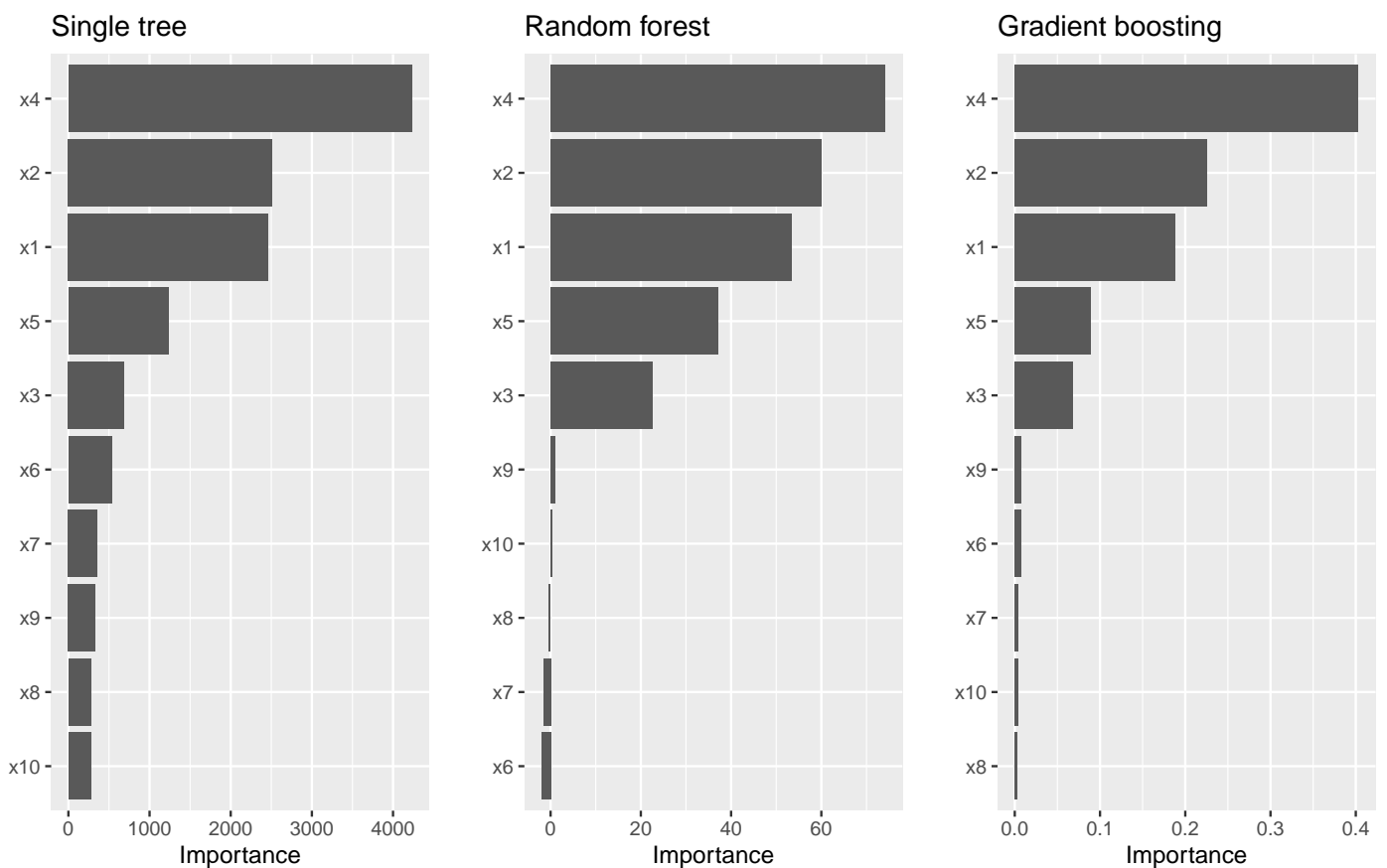

Figure 1: Model-specific VIPs for the three different tree-based models fit to the simulated Friedman data. 
As we would expect, all three methods rank the variables $x 1-\times 5$ as more important than the others. While this is good news, it is unfortunate that we have to remember the different functions and ways of extracting and plotting VI scores from various model fitting functions. This is one place where vip can help... one function to rule them all! Once vip is loaded, we can use vi() to extract a tibble of VI scores.

\# Load required packages
library(vip)

\# Compute model-specific VI scores

vi(tree) \# CART-like decision tree

\#> \# A tibble: $10 \times 2$

\#> Variable Importance

\#> <chr> <dbl>

\#> $1 \times 4 \quad 4234$.

\#> $2 \times 2 \quad 2513$.

\#> $3 \times 1 \quad 2461$.

\#> $4 \times 5 \quad 1230$.

\#> $5 \times 3 \quad 688$.

\#> $6 \times 6 \quad 533$.

\#> $7 \times 7 \quad 357$.

\#> $8 \times 9 \quad 331$.

\#> $9 \times 8 \quad 276$.

\#> $10 \times 10 \quad 275$.

$v i(r f o) \quad \# R F$

\#> \# A tibble: $10 \times 2$

\# Variable Importance

\#> <chr> <dbl>

\#> $1 \times 4 \quad 74.2$

\#> $2 \times 2 \quad 59.9$

\#> $3 \times 1 \quad 53.3$

\#> $4 \times 5 \quad 37.1$

\#> $5 \times 3 \quad 22.5$

\#> $6 \times 9 \quad 1.05$

\#> $7 \times 10 \quad 0.254$

\#> $8 \times 8 \quad-0.408$

\#> $9 \times 7 \quad-1.56$

\#> $10 \times 6 \quad-2.00$

vi(bst) \# GBM

\#> \# A tibble: $10 \times 2$

\# Variable Importance

\#> <chr> <dbl>

\# $\begin{array}{lll}1 \times 4 & 0.403\end{array}$

\#> $2 \times 2 \quad 0.225$

\#> $3 \times 1 \quad 0.189$

\#> $4 \times 5 \quad 0.0894$

\#> $5 \times 3 \quad 0.0682$

\#> $6 \times 9 \quad 0.00802$

\#> $7 \times 6 \quad 0.00746$

\#> $8 \times 7 \quad 0.00400$

\#> $9 \times 10 \quad 0.00377$

\#> $10 \times 8 \quad 0.00262$

Notice how the vi () function always returns a tibble ${ }^{6}$ with two columns: Variable and Importance (the exceptions are coefficient-based models which also include a Sign column giving the sign of the corresponding coefficient, and permutation importance involving multiple Monte Carlo simulations,

\footnotetext{
${ }^{5}$ In order to avoid deprecation warnings due to recent updates to tibble and ggplot2, the code examples in this article are based on the latest development versions of both vip (version 0.2.2.9000) and pdp (version 0.7.0.9000); the URL to the development version of each package is available on its associated CRAN landing page.

${ }^{6}$ Technically, it's a tibble with an additional "vi" class.
} 
but more on that later). Also, by default, vi() always orders the VI scores from highest to lowest; this, among other options, can be controlled by the user (see ?vip: : vi for details). Plotting VI scores with $\operatorname{vip}()$ is just as straightforward. For example, the following code can be used to reproduce Figure 1.

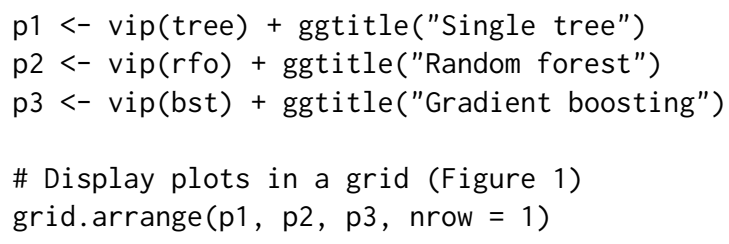

Notice how the $\operatorname{vip}()$ function always returns a "ggplot" object (by default, this will be a bar plot). For large models with many features, a Cleveland dot plot is more effective (in fact, a number of useful plotting options can be fiddled with). Below we call vip() and change a few useful options (the resulting plot is displayed in Figure 2). Note that we can also call vip() directly on a "vi" object if it's already been constructed.
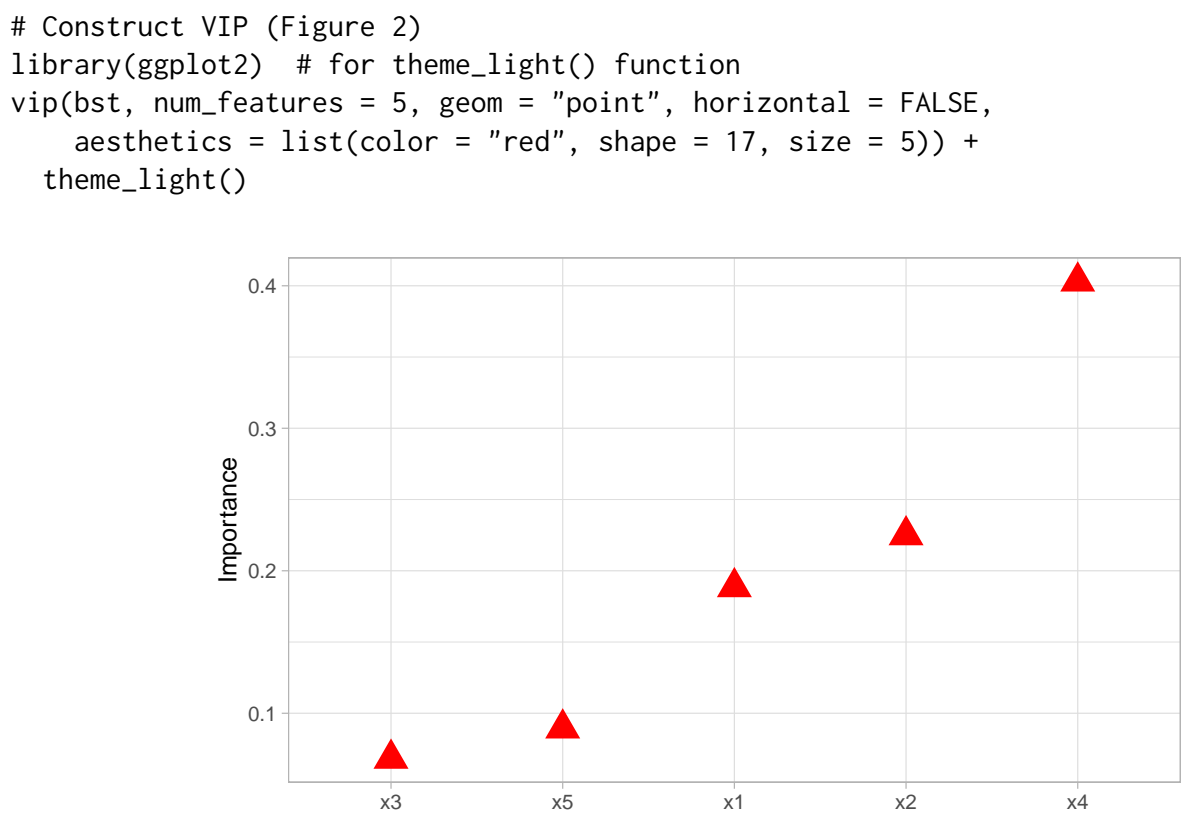

Figure 2: Illustrating various plotting options.

\section{Linear models}

In multiple linear regression, or linear models (LMs), the absolute value of the $t$-statistic (or some other scaled variant of the estimated coefficients) is commonly used as a measure of VI. ${ }^{7}$ The same idea also extends to generalized linear models (GLMs). In the code chunk below, we fit an LM to the simulated Friedman data (trn) allowing for all main effects and two-way interactions, then use the step() function to perform backward elimination. The resulting VIP is displayed in Figure 3.

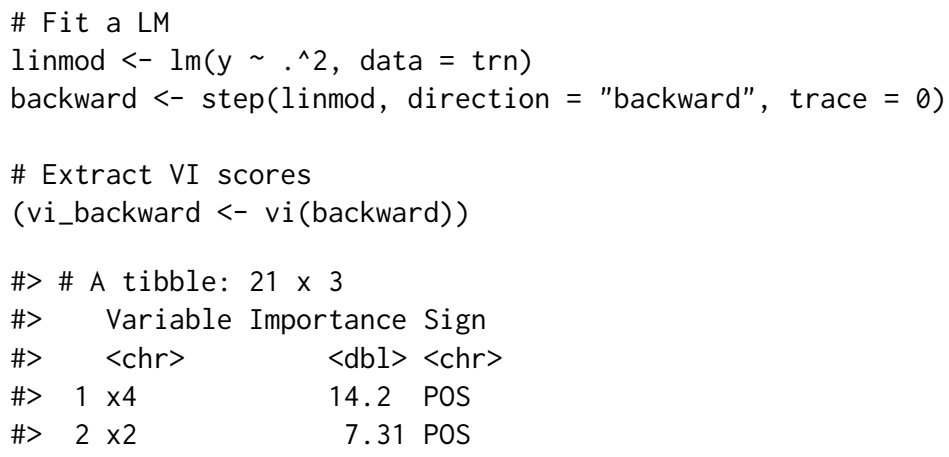

\footnotetext{
${ }^{7}$ Since this approach is biased towards large-scale features it is important to properly standardize the predictors (before fitting the model) or the estimated coefficients.
} 


$\begin{array}{lrlr}\text { \#> } & 3 \times 1 & 5.63 & \text { POS } \\ \text { \#> } & 4 \times 5 & 5.21 \text { POS } \\ \text { \#> } & 5 \times 3: \times 5 & 2.46 & \text { POS } \\ \text { \#> } & 6 \times 1: \times 10 & 2.41 & \text { NEG } \\ \text { \#> } & 7 \times 2: \times 6 & 2.41 \mathrm{NEG} \\ \text { \# } 8 \times 1: \times 5 & 2.37 \mathrm{NEG} \\ \text { \#> } 9 \times 10 & 2.21 \mathrm{POS} \\ \text { \#> } 10 \times 3: \times 4 & 2.01 \mathrm{NEG} \\ \text { \#> \# ... with } 11 \text { more rows }\end{array}$

\# Plot VI scores; by default, 'vip()' displays the top ten features pal <- palette.colors(2, palette $=$ "Okabe-Ito") \# colorblind friendly palette $\operatorname{vip}($ vi_backward, num_features = length $(\operatorname{coef}($ backward $))$, \# Figure 3 geom $=$ "point", horizontal $=$ FALSE, mapping $=$ aes $(\operatorname{color}=$ Sign $))+$ scale_color_manual $($ values $=$ unname $(\mathrm{pal}))+$ theme_light ()$+$ theme (axis.text. $x=$ element_text $($ angle $=45$, hjust $=1)$ )

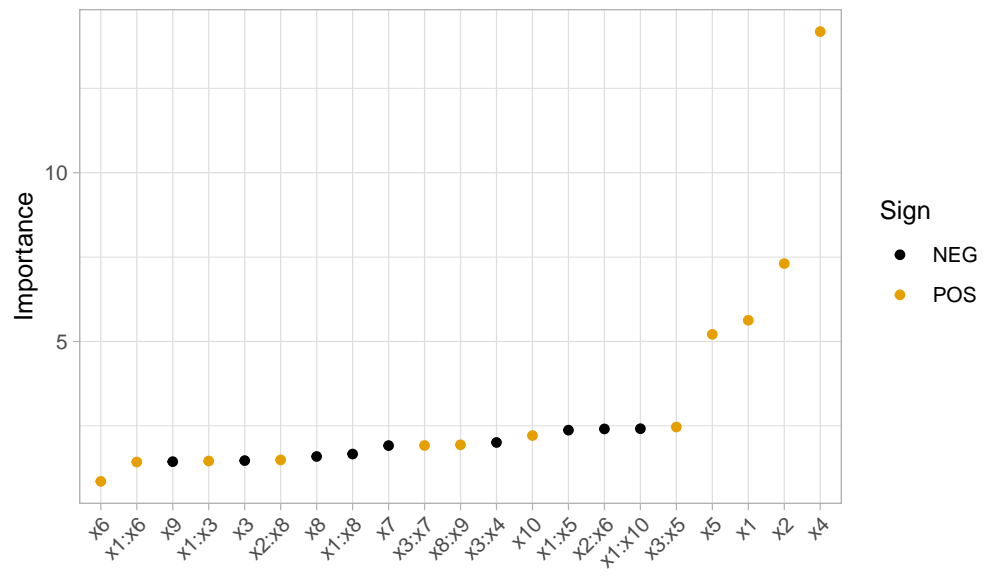

Figure 3: Example VIP from a linear model fit to the simulated Friedman data. The points are colored according to the sign of the associated coefficient.

A major limitation of this approach is that a VI score is assigned to each term in the model, rather than to each individual feature! We can solve this problem using one of the model-agnostic approaches discussed later.

Multivariate adaptive regression splines (MARS), which were introduced in Friedman (1991), is an automatic regression technique and can be seen as a generalization of LMs and GLMs. In the MARS algorithm, the contribution (or VI score) for each predictor is determined using a generalized cross-validation (GCV) statistic (though, other statistics can also be used; see ?vip: :vi_model for details). An example using the earth package (Milborrow, 2019) is given below (the results are plotted in Figure 4):

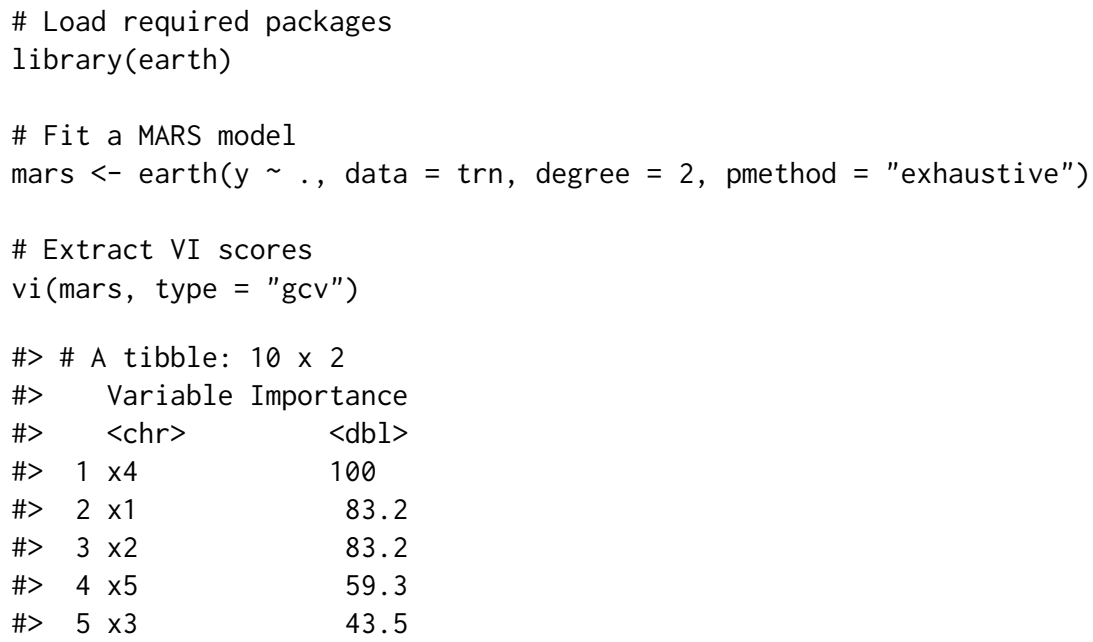




$\begin{array}{lcc}\text { \#> } 6 \times 6 & 0 \\ \#>\quad 7 \times 7 & 0 \\ \#>\quad 8 \times 8 & 0 \\ \#>\quad 9 \times 9 & 0 \\ \#>10 \times 10 & 0 \\ \text { \# Plot VI scores (Figure 4) } \\ \text { vip(mars) }\end{array}$

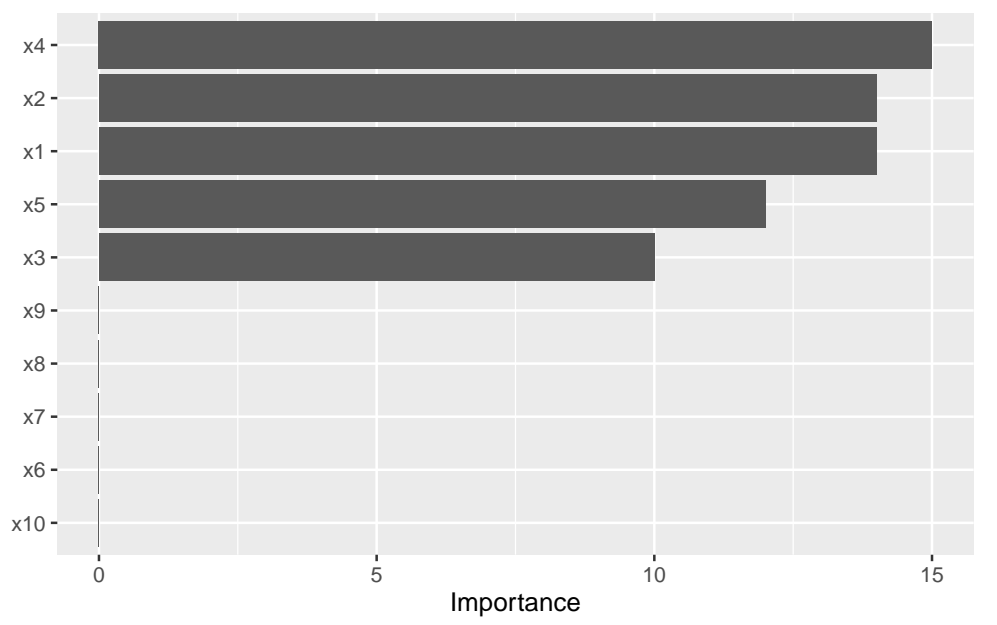

Figure 4: Example VIP from a MARS model fit to the simulated Friedman data.

To access VI scores directly in earth, you can use the earth: : evimp() function.

\section{Neural networks}

For neural networks (NNs), two popular methods for constructing VI scores are the Garson algorithm (Garson, 1991), later modified by Goh (1995), and the Olden algorithm (Olden et al., 2004). For both algorithms, the basis of these VI scores is the network's connection weights. The Garson algorithm determines VI by identifying all weighted connections between the nodes of interest. Olden's algorithm, on the other hand, uses the products of the raw connection weights between each input and output neuron and sums these products across all hidden neurons. This has been shown to outperform the Garson method in various simulations. For DNNs, a similar method due to Gedeon (1997) considers the weights connecting the input features to the first two hidden layers (for simplicity and speed); but this method can be slow for large networks. We illustrate these two methods below using $\operatorname{vip}($ ) with the nnet package (Ripley, 2016) (see the results in Figure 5).

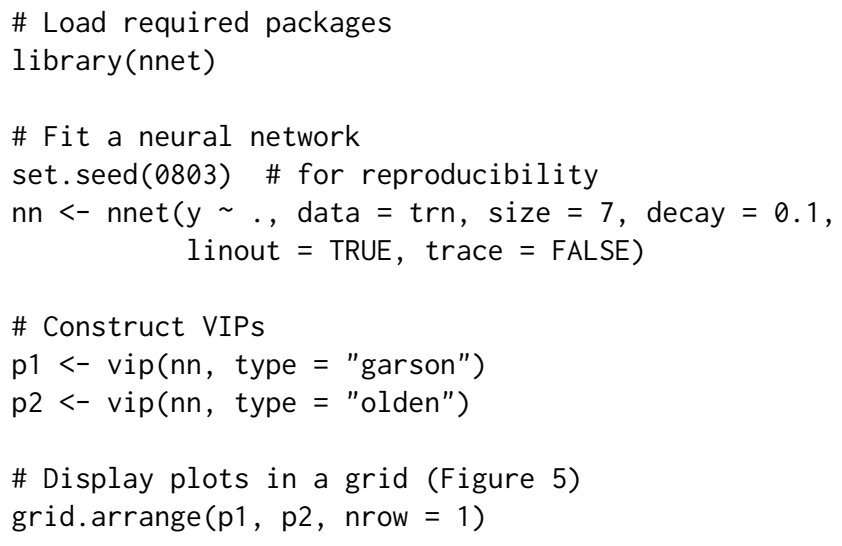

\section{Model-agnostic VI}

Model-agnostic interpretability separates interpretation from the model. Compared to model-specific approaches, model-agnostic VI methods are more flexible and can be applied to any supervised 

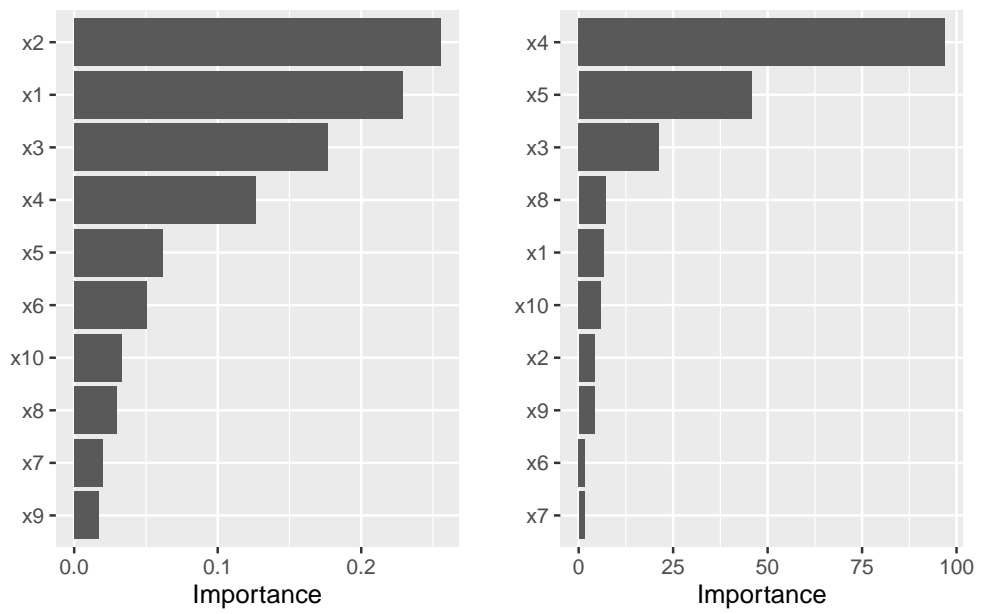

Figure 5: Example VIPs from a single-hidden-layer NN fit to the simulated Friedman data.

learning algorithm. In this section, we discuss model-agnostic methods for quantifying global feature importance using three different approaches: 1) a simple variance-based approach, 2) permutationbased feature importance, and 3) Shapley-based feature importance.

\section{Variance-based methods}

Our first model-agnostic method is based on a simple feature importance ranking measure (FIRM); for details, see Greenwell et al. (2018), Zien et al. (2009), and Scholbeck et al. (2019). The specific approach used here is based on quantifying the "flatness" of the effects of each feature. ${ }^{8}$ Feature effects can be assessed using partial dependence plots (PDPs) (Friedman, 2001) or individual conditional expectation (ICE) curves (Goldstein et al., 2015). PDPs and ICE curves help visualize the effect of low cardinality subsets of the feature space on the estimated prediction surface (e.g., main effects and two/threeway interaction effects.). They are also model-agnostic and can be constructed in the same way for any supervised learning algorithm. Below, we fit a projection pursuit regression (PPR) model (see ?stats: : ppr for details and references) and construct PDPs for each feature using the pdp package Greenwell (2017). The results are displayed in Figure 6. Notice how the PDPs for the uninformative features are relatively flat compared to the PDPs for features $x 1-x 5$ !
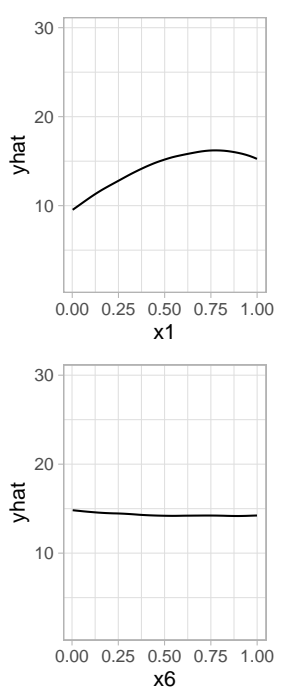

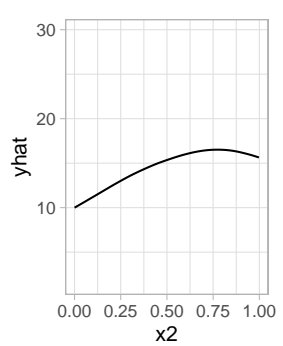

$\mathrm{x} 2$

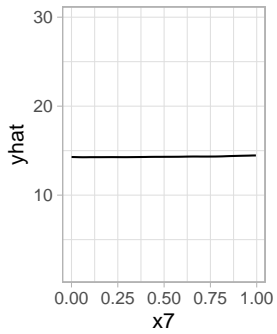

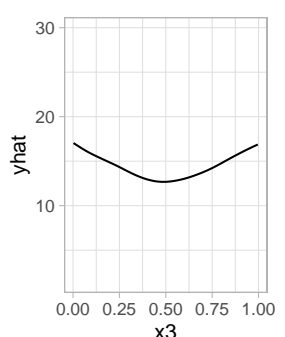

$\times 3$

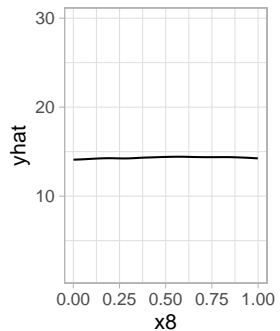

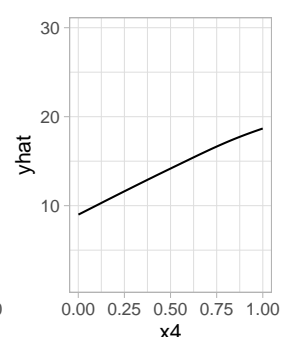

$\mathrm{x} 4$

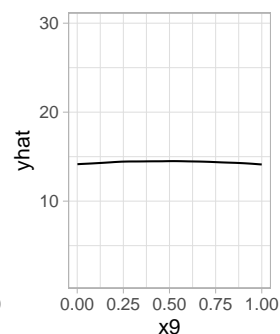

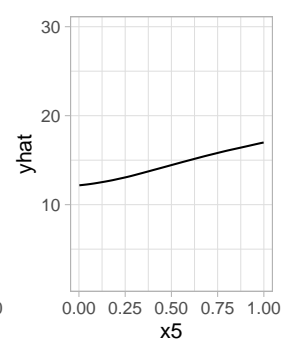

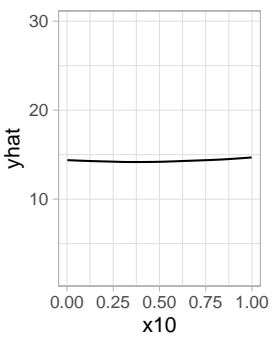

Figure 6: PDPs of main effects in the PPR model fit to the simulated Friedman data.

Next, we compute PDP-based VI scores for the fitted PPR and NN models. The PDP method constructs VI scores that quantify the relative "flatness" of each PDP (by default, this is defined by computing the standard deviation of the $y$-axis values for each PDP). To use the PDP method, specify method $=$ "firm" in the call to $v i()$ or $\operatorname{vip}()$ (or just use vi_firm() directly):

\footnotetext{
${ }^{8} \mathrm{~A}$ similar approach is taken in the vivo package (Kozak and Biecek, 2019).
} 

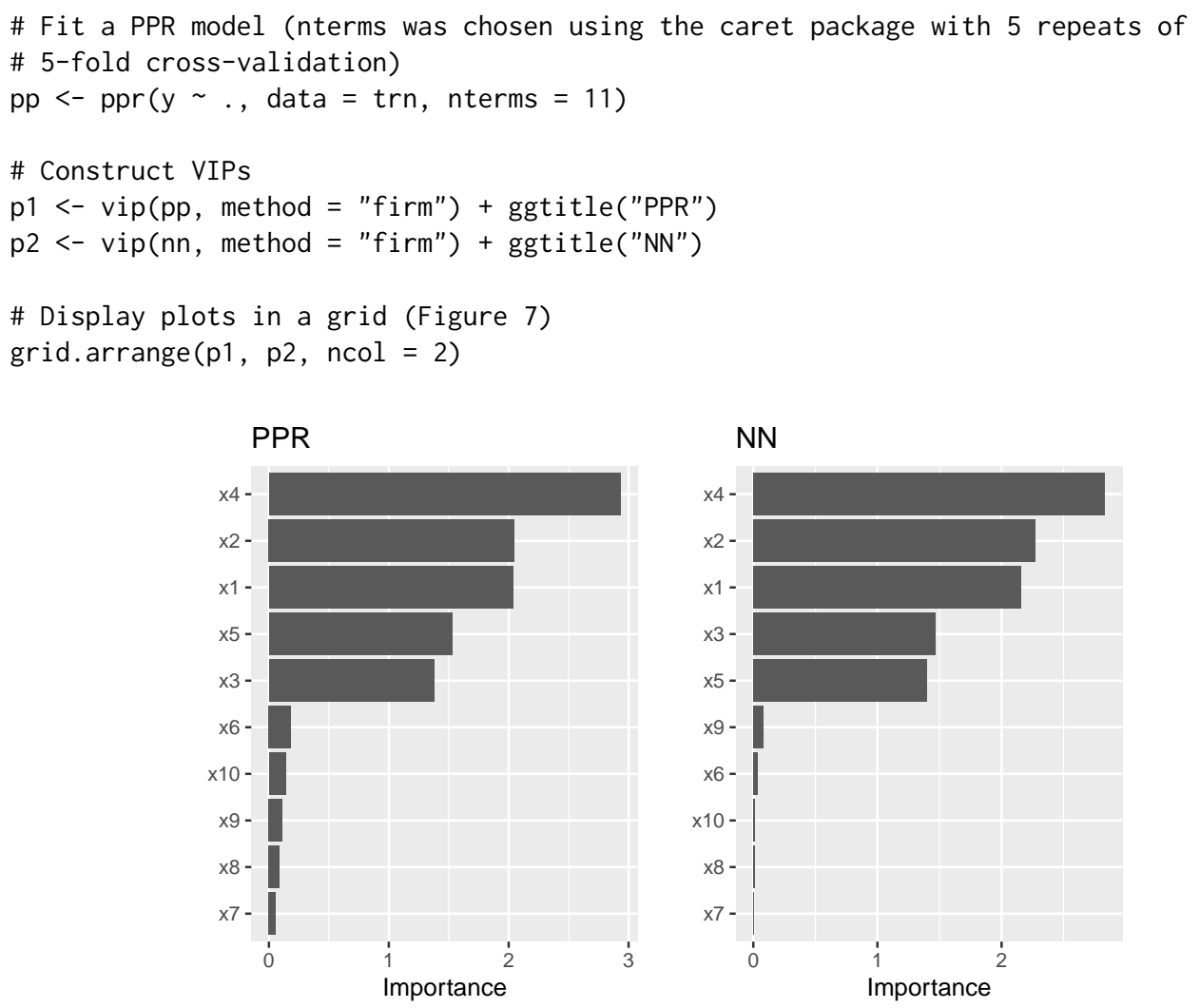

Figure 7: PDP-based feature importance for the PPR and NN models fit to the simulated Friedman data.

In Figure 7 we display the PDP-based feature importance for the previously obtained PPR and NN models. These VI scores essentially capture the variability in the partial dependence values for each main effect.

The ICE curve method is similar to the PDP method, except that we measure the "flatness" of each individual ICE curve and then aggregate the results (e.g., by averaging). If there are no (substantial) interaction effects, using ICE curves will produce results similar to using PDPs (which are just averaged ICE curves). However, if strong interaction effects are present, they can obfuscate the main effects and render the PDP-based approach less useful (since the PDPs for important features can be relatively flat when certain interactions are present; see Goldstein et al. (2015) for details). In fact, it is probably safest to always use ICE curves when employing the FIRM method.

Below, we display the ICE curves for each feature in the fitted PPR model using the same $y$-axis scale; see Figure 8. Again, there is a clear difference between the ICE curves for features $x 1-x 5$ and $x 6-x 10$; the later being relatively flat by comparison. Also, notice how the ICE curves within each feature are relatively parallel (if the ICE curves within each feature were perfectly parallel, the standard deviation for each curve would be the same and the results will be identical to the PDP method). In this example, the interaction term between $x 1$ and $x 2$ does not obfuscate the PDPs for the main effects and the results are not much different.

Obtaining the ICE-based feature importance scores is also straightforward, just specify ice = TRUE when using the FIRM approach. This is illustrated in the code chunk below and the results, which are displayed in Figure 9, are similar to those obtained using the PDP method.

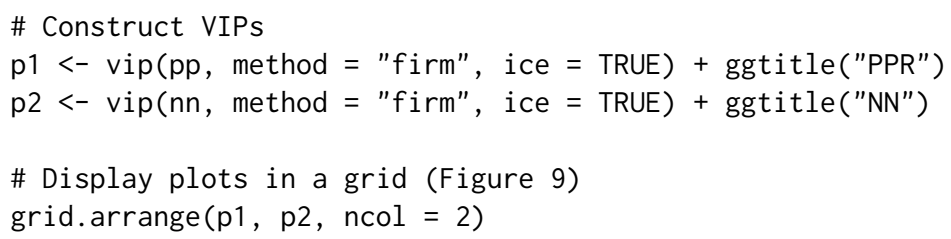

When using method = "firm", the feature effect values are stored in an attribute called "effects". This is a convenience so that the feature effect plots (e.g., PDPs and ICE curves) can easily be reconstructed and compared with the VI scores, as demonstrated in the example below (see Figure 10): 

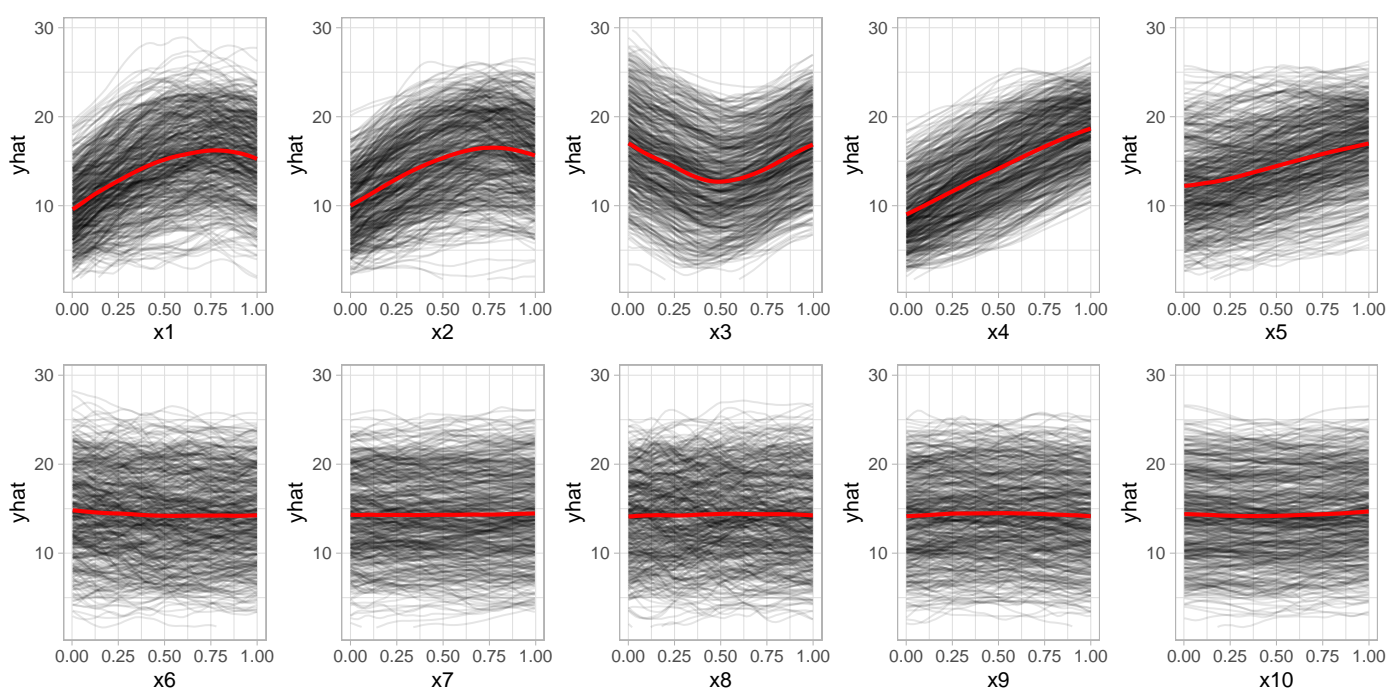

Figure 8: ICE curves for each feature in the PPR model fit to the simulated Friedman data. The red curve represents the PDP (i.e., the averaged ICE curves).
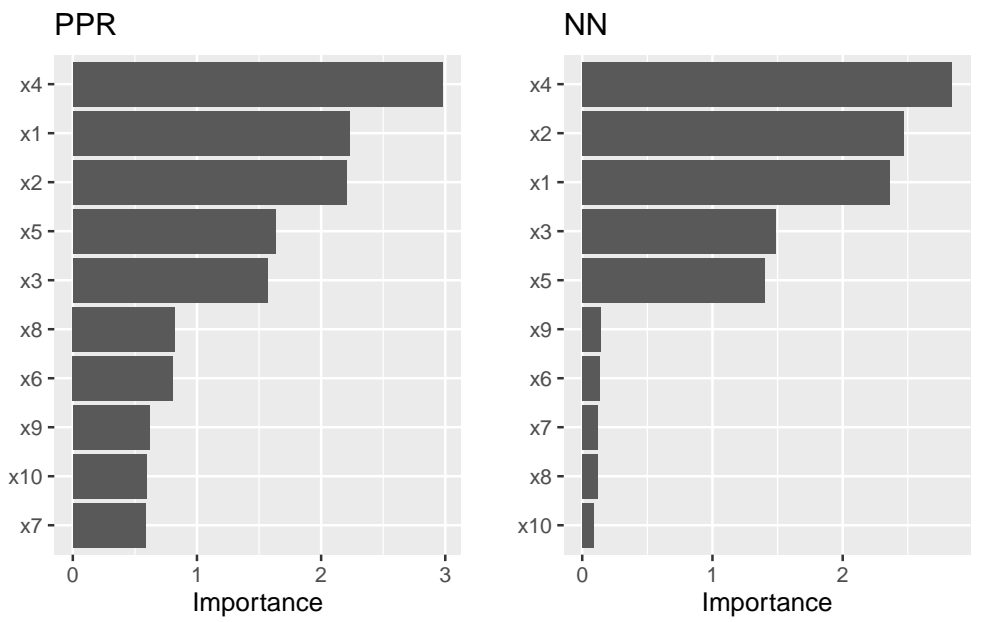

Figure 9: ICE-based feature importance for the PPR and NN models fit to the simulated Friedman data.

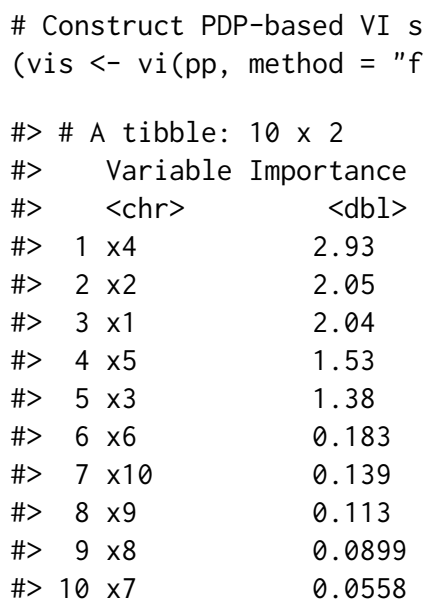

\# Reconstruct PDPs for all 10 features (Figure 10)

$\operatorname{par}(\mathrm{mfrow}=\mathrm{c}(2,5))$

for (name in paste0("x", 1:10)) \{

$\operatorname{plot}(\operatorname{attr}(v i s$, which $="$ effects") $[[$ name $]]$, type $=" 1 ", y \lim =c(9,19)$, las $=1)$ \} 

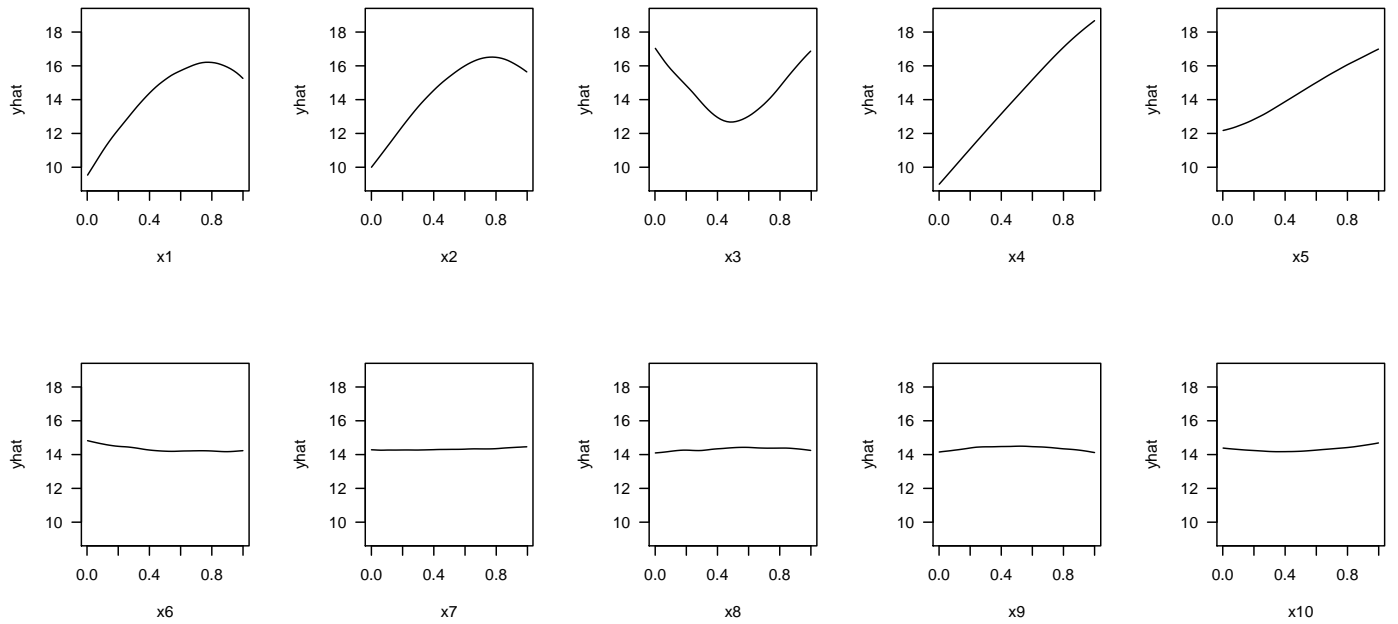

Figure 10: PDPs for all ten features reconstructed from the pdp attribute of the vis object.

\section{Permutation method}

The permutation method exists in various forms and was made popular in Breiman (2001) for RFs, before being generalized and extended in Fisher et al. (2018). The permutation approach used in vip is quite simple and is outlined in Algorithm 1 below. The idea is that if we randomly permute the values of an important feature in the training data, the training performance would degrade (since permuting the values of a feature effectively destroys any relationship between that feature and the target variable). This of course assumes that the model has been properly tuned (e.g., using cross-validation) and is not over fitting. The permutation approach uses the difference between some baseline performance measure (e.g., training $R^{2}, \mathrm{AUC}$, or RMSE) and the same performance measure obtained after permuting the values of a particular feature in the training data (Note: the model is NOT refit to the training data after randomly permuting the values of a feature). It is also important to note that this method may not be appropriate when you have, for example, highly correlated features (since permuting one feature at a time may lead to unlikely data instances).

Let $X_{1}, X_{2}, \ldots, X_{j}$ be the features of interest and let $\mathcal{M}_{\text {orig }}$ be the baseline performance metric for the trained model; for brevity, we'll assume smaller is better (e.g., classification error or RMSE). The permutation-based importance scores can be computed as follows:

1. For $i=1,2, \ldots, j$ :

(a) Permute the values of feature $X_{i}$ in the training data.

(b) Recompute the performance metric on the permuted data $\mathcal{M}_{\text {perm }}$.

(c) Record the difference from baseline using imp $\left(X_{i}\right)=\mathcal{M}_{\text {perm }}-\mathcal{M}_{\text {orig }}$.

2. Return the VI scores $\operatorname{imp}\left(X_{1}\right), \operatorname{imp}\left(X_{2}\right), \ldots, \operatorname{imp}\left(X_{j}\right)$.

Algorithm 1: A simple algorithm for constructing permutation-based VI scores.

Algorithm 1 can be improved or modified in a number of ways. For instance, the process can be repeated several times and the results averaged together. This helps to provide more stable VI scores, and also the opportunity to measure their variability. Rather than taking the difference in step (c), Molnar (2019b, sec. 5.5.4) argues that using the ratio $\mathcal{M}_{\text {perm }} / \mathcal{M}_{\text {orig }}$ makes the importance scores more comparable across different problems. It's also possible to assign importance scores to groups of features (e.g., by permuting more than one feature at a time); this would be useful if features can be categorized into mutually exclusive groups, for instance, categorical features that have been one-hot-encoded.

To use the permutation approach in vip, specify method = "permute" in the call to vi() or vip() (or you can use vi_permute() directly). Note that using method = "permute" requires specifying a few additional arguments (e.g., the training data, target name or vector of target values, a prediction function, etc.); see ?vi_permute for details.

An example is given below for the previously fitted PPR and NN models. Here we use $R^{2}$ (metric $=$ "rsquared") as the evaluation metric. The results, which are displayed in Figure 11, agree with 
those obtained using the PDP- and ICE-based methods.
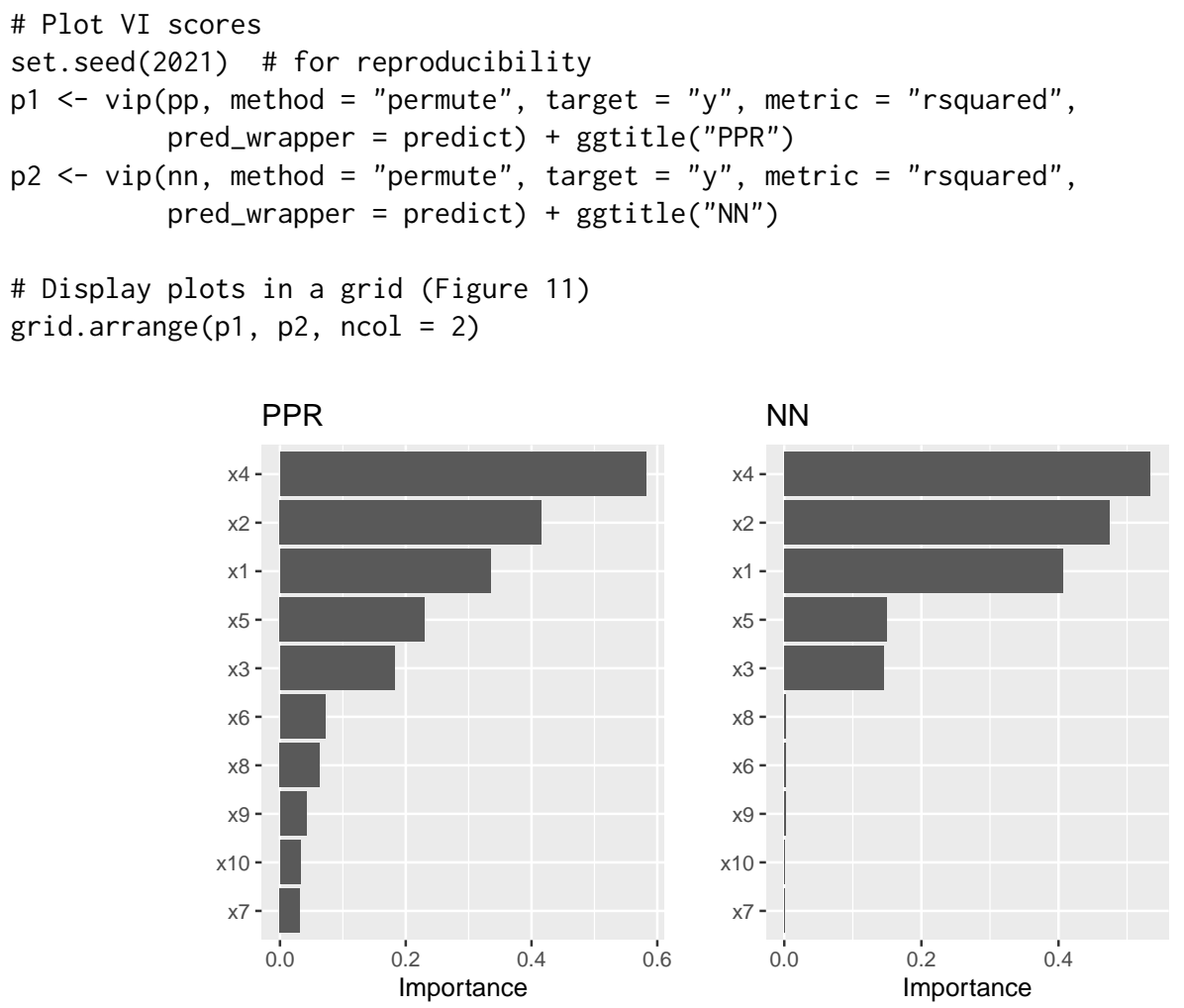

Figure 11: Permutation-based feature importance for the PPR and NN models fit to the simulated Friedman data.

The permutation approach introduces randomness into the procedure and therefore should be run more than once if computationally feasible. The upside to performing multiple runs of Algorithm 1 is that it allows us to compute standard errors (among other metrics) for the estimated VI scores, as illustrated in the example below; here we specify nsim $=10$ to request that each feature be permuted 10 times and the results averaged together. (Additionally, if nsim $>1$, you can set geom = "boxplot" in the call to $\operatorname{vip}($ ) to construct boxplots of the raw permutation-based VI scores. This is useful if you want to visualize the variability in each of the VI estimates; see Figure 12 for an example.)

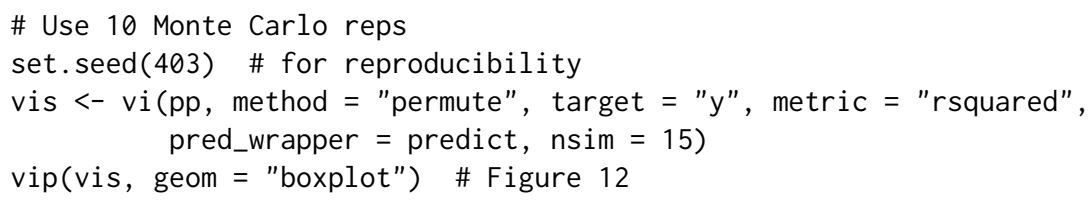

All available performance metrics for regression and classification can be listed using the list_metrics() function, for example:

\begin{tabular}{|c|c|c|c|c|}
\hline \#> & Metric & Description & & Task \\
\hline$\#>1$ & accuracy & Classification accuracy & Binary/multiclass & classification \\
\hline$\#>2$ & error & Misclassification error & Binary/multiclass & classification \\
\hline$\#>3$ & auc & Area under (ROC) curve & Binary & classification \\
\hline$\#>4$ & logloss & Log loss & Binary & classification \\
\hline$\#>5$ & mauc Multiclass & s area under (ROC) curve & Multiclass & classification \\
\hline$\#>6$ & mae & Mean absolute error & & Regression \\
\hline$\#>7$ & mse & Mean squared error & & Regression \\
\hline$\#>8$ & $r 2$ & R squared & & Regression \\
\hline \#> 9 & rsquared & R squared & & Regression \\
\hline \#> 10 & rmse & Root mean squared error & & Regression \\
\hline \#> 11 & sse & Sum of squared errors & & Regression \\
\hline
\end{tabular}




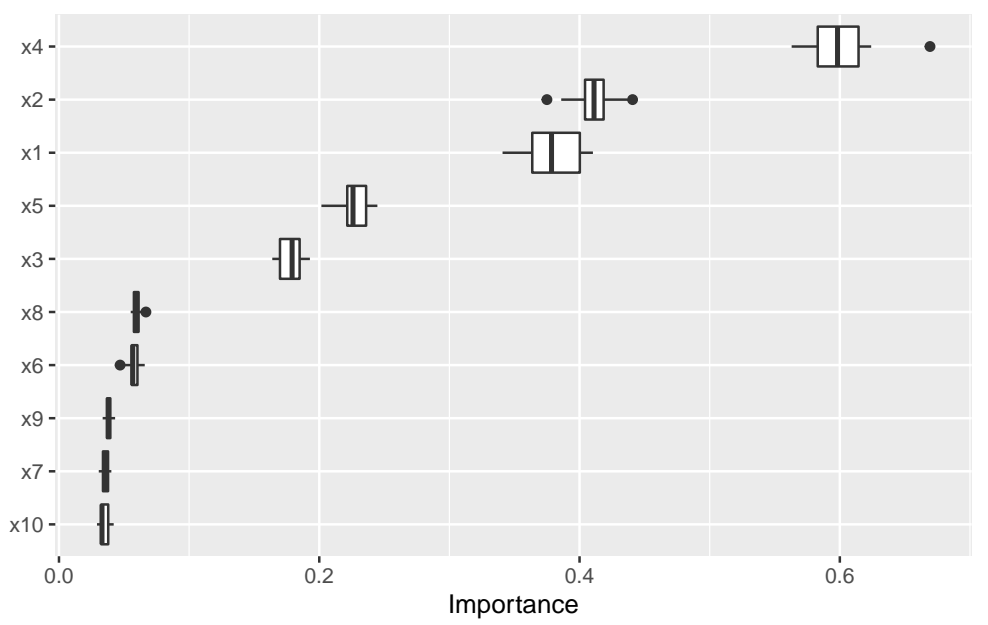

Figure 12: Boxplots of VI scores using the permutation method with 15 Monte Carlo repetitions.

We can also use a custom metric (i.e., loss function). Suppose for example you want to measure importance using the mean absolute error (MAE):

$$
M A E=\frac{1}{n} \sum_{i=1}^{n}\left|Y_{i}-\widehat{f}\left(\boldsymbol{X}_{i}\right)\right|
$$

where $\widehat{f}\left(\boldsymbol{X}_{i}\right)$ is the predicted value of $Y_{i}$. A simple function implementing this metric is given below (note that, according to the documentation in ?vi_permute, user-supplied metric functions require two arguments: actual and predicted).

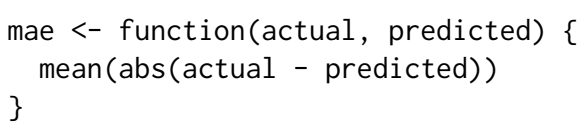

To use this for computing permutation-based VI scores just pass it via the metric argument (be warned, however, that the metric used for computing permutation importance should be the same as the metric used to train and tune the model). Also, since this is a custom metric, we need to specify whether a smaller value indicates better performance by setting smaller_is_better $=$ TRUE. The results, which are displayed in Figure 13, are similar to those in Figure 11, albeit a different scale.

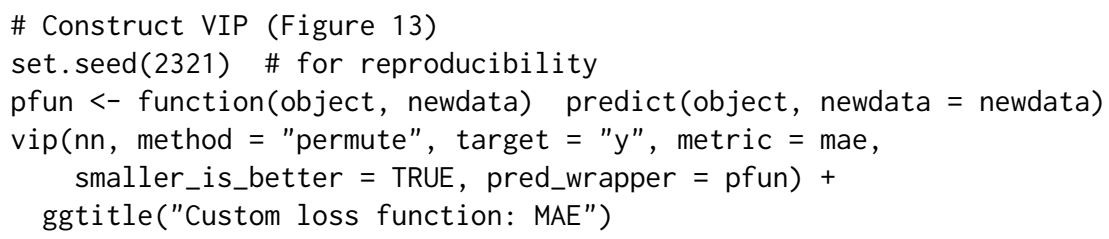

Although permutation importance is most naturally computed on the training data, it may also be useful to do the shuffling and measure performance on new data! This is discussed in depth in Molnar (2019b, sec. 5.2). For users interested in computing permutation importance using new data,

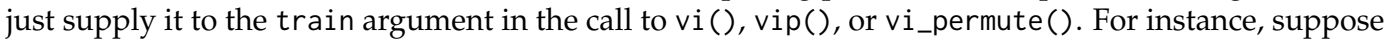
we wanted to only use a fraction of the original training data to carry out the computations. In this case, we could simply pass the sampled data to the train argument as follows:

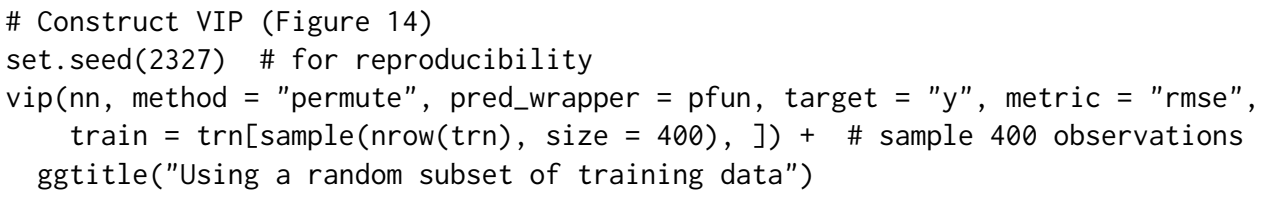

When using the permutation method with nsim $>1$, the default is to keep all the permutation scores as an attribute called "raw_scores"; you can turn this behavior off by setting keep = FALSE in the call to vi_permute(), vi(), or vip(). If keep = TRUE and nsim $>1$, you can request all permutation scores to be plotted by setting all_permutation = TRUE in the call to vip(), as demonstrated in the code chunk below (see Figure 15). This also let's you visually inspect the variability in the permutation scores within each feature. 


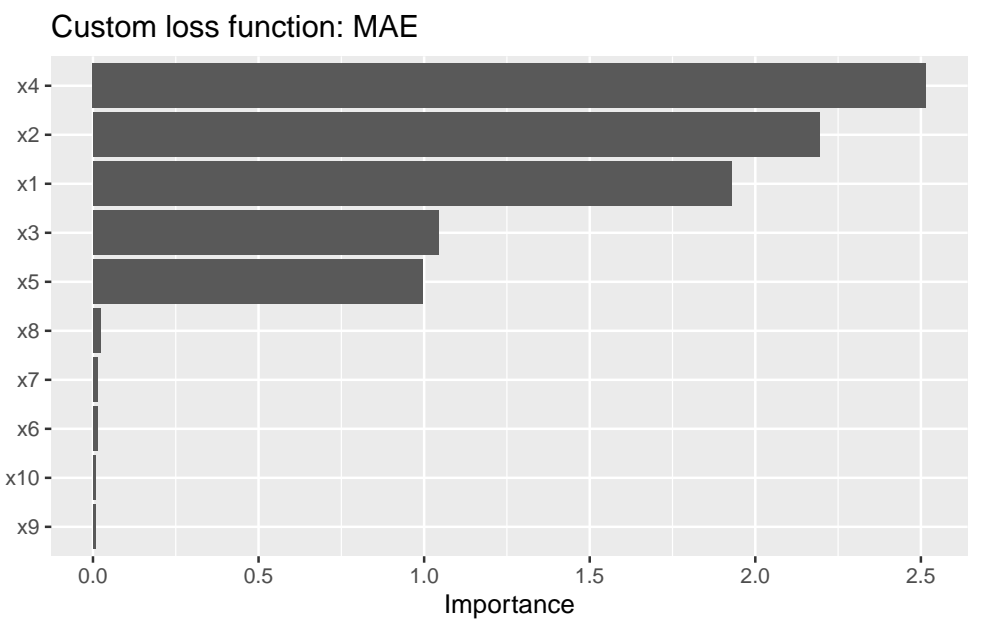

Figure 13: Permutation-based VI scores for the NN model fit to the simulated Friedman data. In this example, permutation importance is based on the MAE metric.

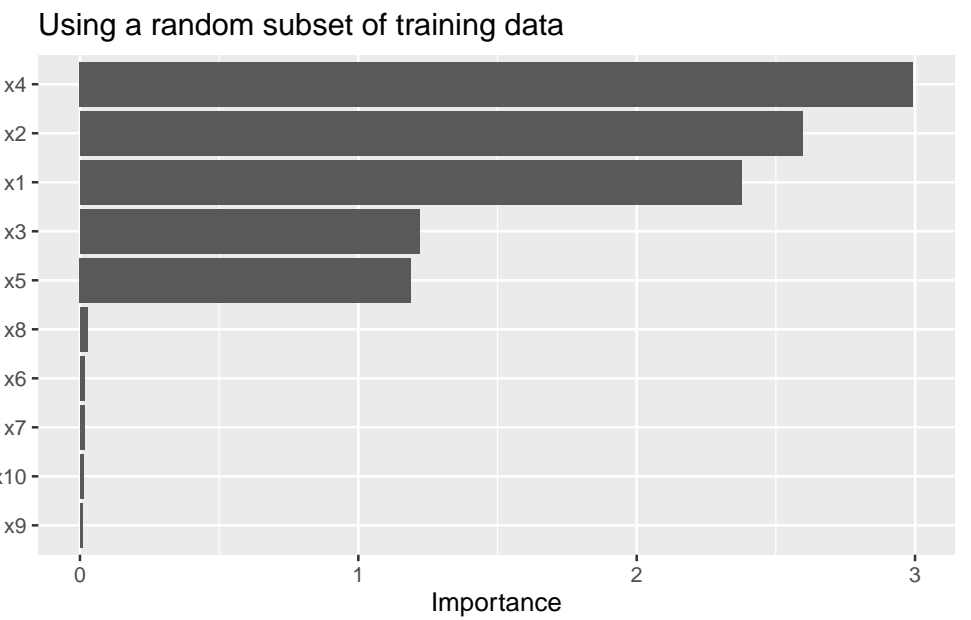

Figure 14: Permutation-based feature importance for the NN model fit to the simulated Friedman data. In this example, permutation importance is based on a random sample of 400 training observations.

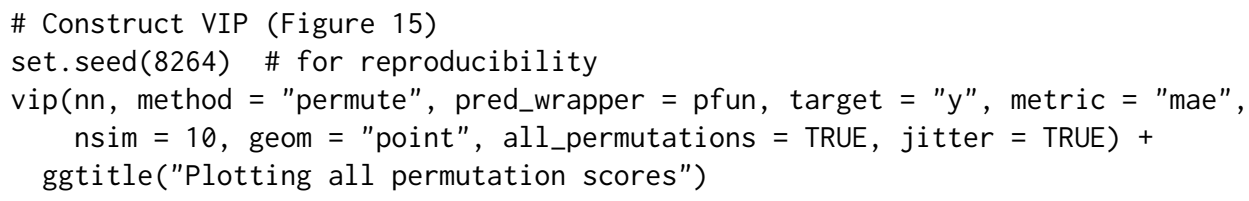

\section{Benchmarks}

In this section, we compare the performance of four implementations of permutation-based VI scores: iml : : FeatureImp() (version 0.10.0), ingredients: : feature_importance() (version 1.3.1), mmpf: : permutationImportance (version 0.0.5), and vip: : vi() (version 0.2.2.9000).

We simulated 10,000 training observations from the Friedman 1 benchmark problem and trained a random forest using the ranger package. For each implementation, we computed permutation-based VI scores 100 times using the microbenchmark package (Mersmann, 2019). For this benchmark we did not use any of the parallel processing capability available in the iml and vip implementations. The results from microbenchmark are displayed in Figure 16 and summarized in the output below. In this case, the vip package (version 0.2.2.9000) was the fastest, followed closely by ingredients and mmpf. It should be noted, however, that the implementations in vip and iml can be parallelized. To the best of our knowledge, this is not the case for ingredients or mmpf (although it would not be difficult to write a simple parallel wrapper for either). The code used to generate these benchmarks can be found at http://bit.1y/2TogXrq. 


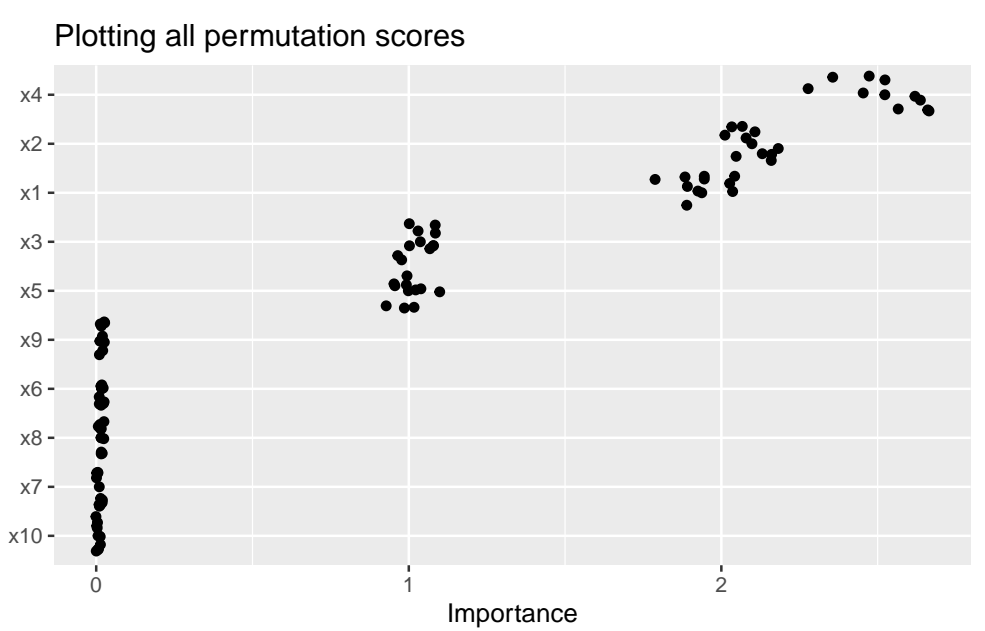

Figure 15: Permutation-based feature importance for the NN model fit to the simulated Friedman data. In this example, all the permutation importance scores (points) are displayed for each feature along with their average (bars).

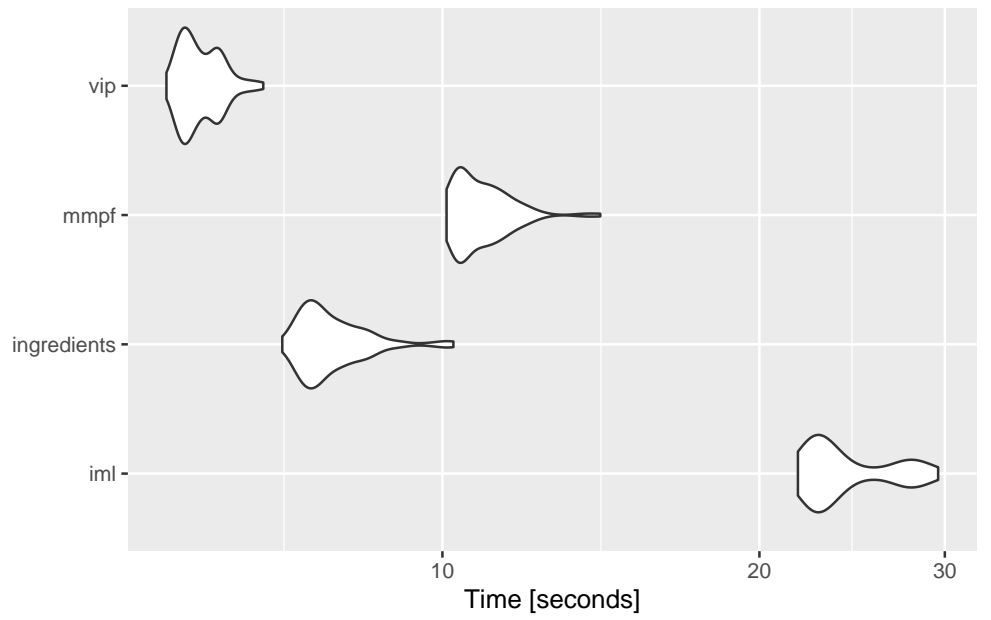

Figure 16: Violin plots comparing the computation time from three different implementations of permutation-based VI scores across 100 simulations.

\section{Shapley method}

Although vip focuses on global VI methods, it is becoming increasing popular to asses global importance by aggregating local VI measures; in particular, Shapley explanations (Štrumbelj and Kononenko, 2014). Using Shapley values (a method from coalitional game theory), the prediction for a single instance $x^{\star}$ can be explained by assuming that each feature value in $x^{\star}$ is a "player" in a game with a payout equal to the corresponding prediction $\widehat{f}\left(x^{\star}\right)$. Shapley values tell us how to fairly distribute the "payout" (i.e., prediction) among the features. Shapley values have become popular due to the attractive fairness properties they posses (Lundberg and Lee, 2017). The most popular implementation is available in the Python shap package (Lundberg and Lee, 2017); although a number of implementations are now available in R; for example, iml, iBreakDown (Biecek et al., 2019b), and fastshap (Greenwell, 2019).

Obtaining a global VI score from Shapley values requires aggregating the Shapley values for each feature across the entire training set (or at least a reasonable sample thereof). In particular, we use the mean of the absolute value of the individual Shapley values for each feature. Unfortunately, Shapley values can be computationally expensive, and therefore this approach may not be feasible for large training sets (say, $>3000$ observations). The fastshap package provides some relief by exploiting a few computational tricks, including the option to perform computations in parallel (see ?fastshap: : explain for details). Also, fast and exact algorithms (Lundberg et al., 2019) can be exploited for certain classes of models.

Starting with vip version 0.2.2.9000 you can now use method = "shap" in the call to vi() (or use vi_shap() directly) to compute global Shapley-based VI scores using the method described above 
(provided you have the fastshap package installed)—see ?vip: :vi_shap for details. To illustrate, we compute Shapley-based VI scores from an xgboost model (Chen et al., 2019) using the Friedman data from earlier; the results are displayed in Figure 17. ${ }^{9}$ (Note: specifying include_type = TRUE in the call to $\operatorname{vip}()$ causes the type of VI computed to be displayed as part of the axis label.)
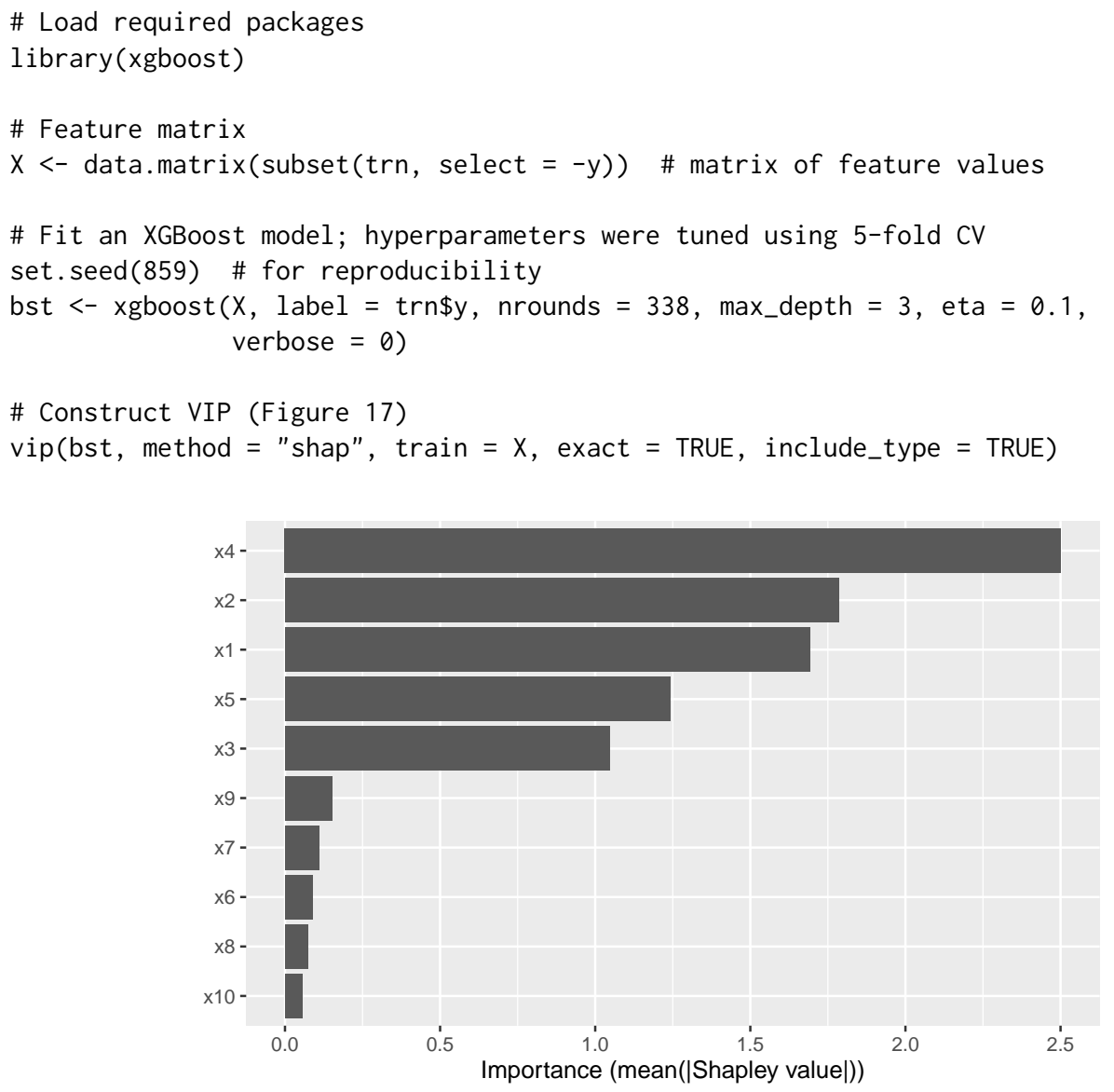

Figure 17: Shapley-based VI scores from an XGBoost model fit to the simulated Friedman data.

\section{Drawbacks of existing methods}

As discussed in Hooker and Mentch (2019), permute-and-predict methods-like PDPs, ICE curves, and permutation importance — can produce results that are highly misleading. ${ }^{10}$ For example, the standard approach to computing permutation-based VI scores involves independently permuting individual features. This implicitly makes the assumption that the observed features are statistically independent. In practice, however, features are often not independent which can lead to nonsensical VI scores. One way to mitigate this issue is to use the conditional approach described in Strobl et al. (2008); Hooker and Mentch (2019) provides additional alternatives, such as permute-and-relearn importance. Unfortunately, to the best of our knowledge, this approach is not yet available for general purpose. A similar modification can be applied to PDPs (Parr and Wilson, 2019) ${ }^{11}$ which seems reasonable to use in the FIRM approach when strong dependencies among the features are present (though, we have not given this much thought or consideration).

We already mentioned that PDPs can be misleading in the presence of strong interaction effects. This drawback, of course, equally applies to the FIRM approach using PDPs for computing VI scores. As discussed earlier, this can be mitigated by using ICE curves instead. Another alternative would be to use accumulated local effect (ALE) plots (Apley and Zhu, 2016) (though we haven't really tested this idea). Compared to PDPs, ALE plots have the advantage of being faster to compute and less affected by strong dependencies among the features. The downside, however, is that ALE plots are

\footnotetext{
${ }^{9}$ Note that the exact $=$ TRUE option is only available if you have fastshap version 0.0 .4 or later

${ }^{10} \mathrm{It}^{\prime} \mathrm{s}$ been argued that approximate Shapley values share the same drawback, however, Janzing et al. (2019) makes a compelling case against those arguments.

${ }^{11} \mathrm{~A}$ basic R implementation is available at https: //github. com/bgreenwell/rstratx.
} 
more complicated to implement (hence, they are not currently available when using method = "firm"). ALE plots are available in the ALEPlot (Apley, 2018) and iml packages.

Hooker (2007) also argues that feature importance (which concern only main effects) can be misleading in high dimensional settings, especially when there are strong dependencies and interaction effects among the features, and suggests an approach based on a generalized functional ANOVA decomposition - though, to our knowledge, this approach is not widely implemented in open source.

\section{Use sparklines to characterize feature effects}

Starting with vip 0.1.3, we have included a new function add_sparklines() for constructing HTMLbased VI tables; however, this feature requires the DT package (Xie et al., 2019). The primary difference between $v i()$ and add_sparklines() is that the latter includes an Effect column that displays a sparkline representation of the partial dependence function for each feature. This is a concise way to display both feature importance and feature effect information in a single (interactive) table. See ?vip: : add_sparklines for details. We illustrate the basic use of add_sparklines() in the code chunk below where we fit a ranger-based random forest using the mlr3 package (Lang et al., 2019). ${ }^{12}$

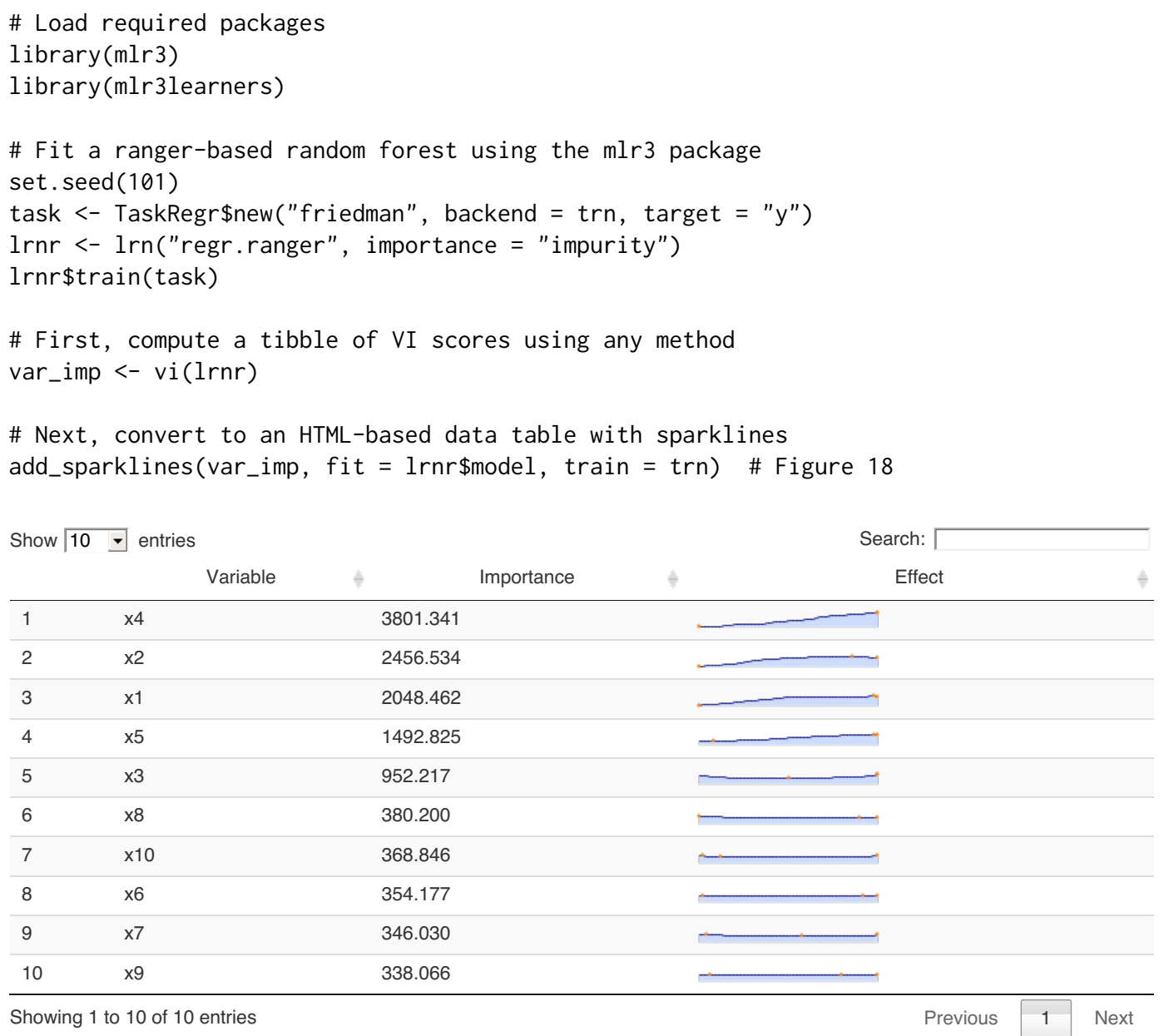

Figure 18: Variable importance scores along with a sparkline representation of feature effects.

\section{Ames housing example}

For illustration, we'll use the Ames housing data (Cock, 2011) which are available in the AmesHousing package (Kuhn, 2017). These data describe the sale of individual residential properties in Ames, Iowa from 2006-2010. The data set contains 2930 observations, 80 features (23 nominal, 23 ordinal, 14 discrete, and 20 continuous), and a continuous target giving the sale price of the home. The version

\footnotetext{
${ }^{12}$ Note: Here we use the ... argument to pass the original training to pdp: : partial(); this is to avoid conflicts caused by mlr3's data.table backend (Dowle and Srinivasan, 2019).
} 
we'll load is a cleaned up version of the original data set and treats all categorical variables as nominal (see ?AmesHousing: : make_ames for details).

Using the R package SuperLearner (Polley et al., 2019), we trained five models using 5-fold crossvalidation: a GBM using the xgboost package, an RF using the ranger package, a MARS model using the earth package, a GLMNET model using the glmnet package (Friedman et al., 2019), and a support vector regression model using the kernlab package (Karatzoglou et al., 2019). The magnitude of the coefficients from the meta learner indicate which models contribute the most (if at all) to new predictions.

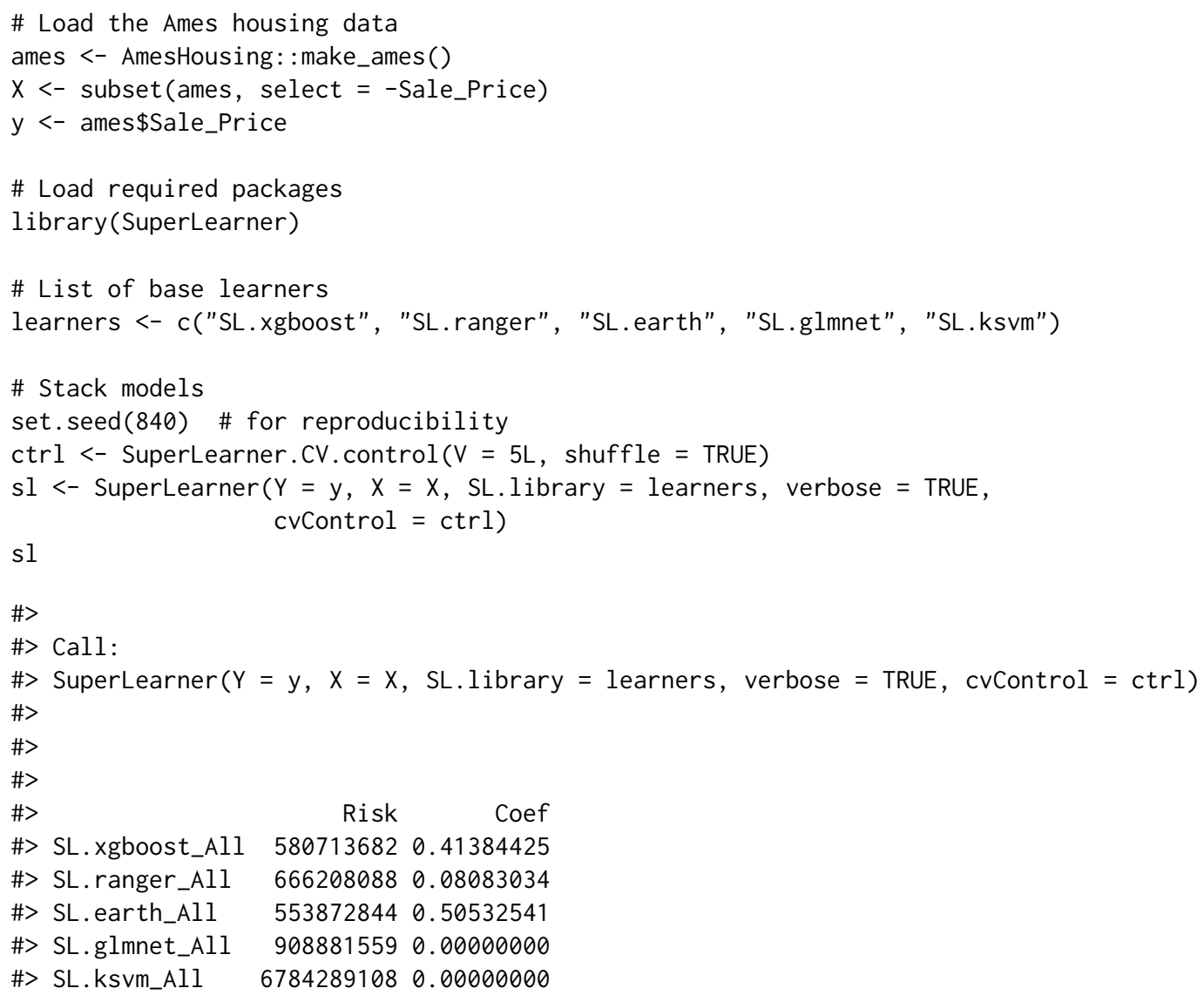

In the code chunks below we request permutation-based VI scores and a sparkline representation of the PDPs for the top ten features. For this we need to define a couple of wrapper functions: one for computing predictions (for the permutation VI scores), and one for computing averaged predictions (for the PDPs).

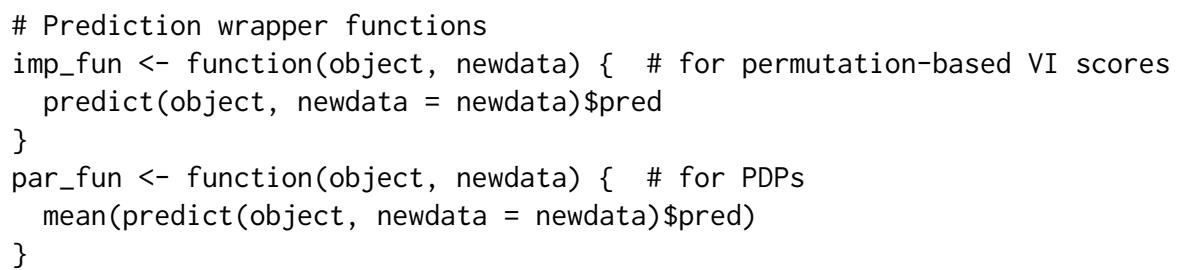

To speed up the process, we perform the computations in parallel by setting parallel = TRUE in the calls to vi() and add_sparklines(). Note that we first need to set up a parallel backend for this to work. Both vip and pdp use plyr (Wickham, 2019)—which relies on foreach—so any parallel backend supported by the foreach package should work. Below we use a socket approach with the doParallel backend (Corporation and Weston, 2019) using a cluster of size five.

\# Setup parallel backend

library(doParallel) \# load the parallel backend

cl <- makeCluster(5) \# use 5 workers

registerDoParallel(cl) \# register the parallel backend

\# Permutation-based feature importance 


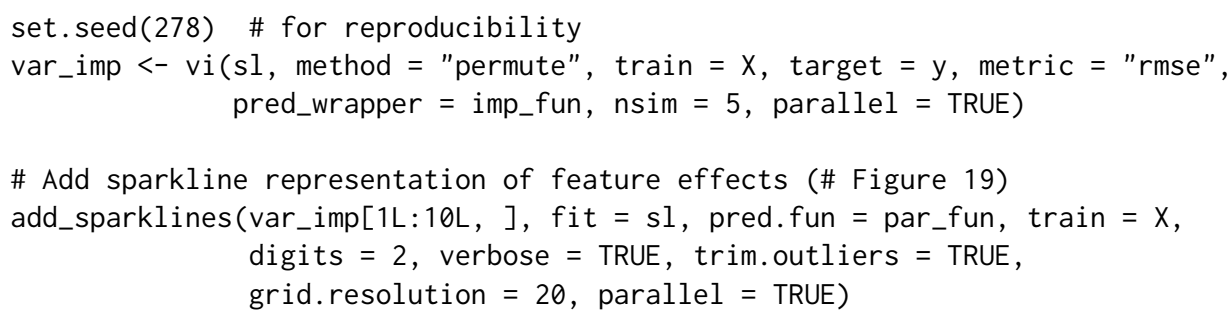

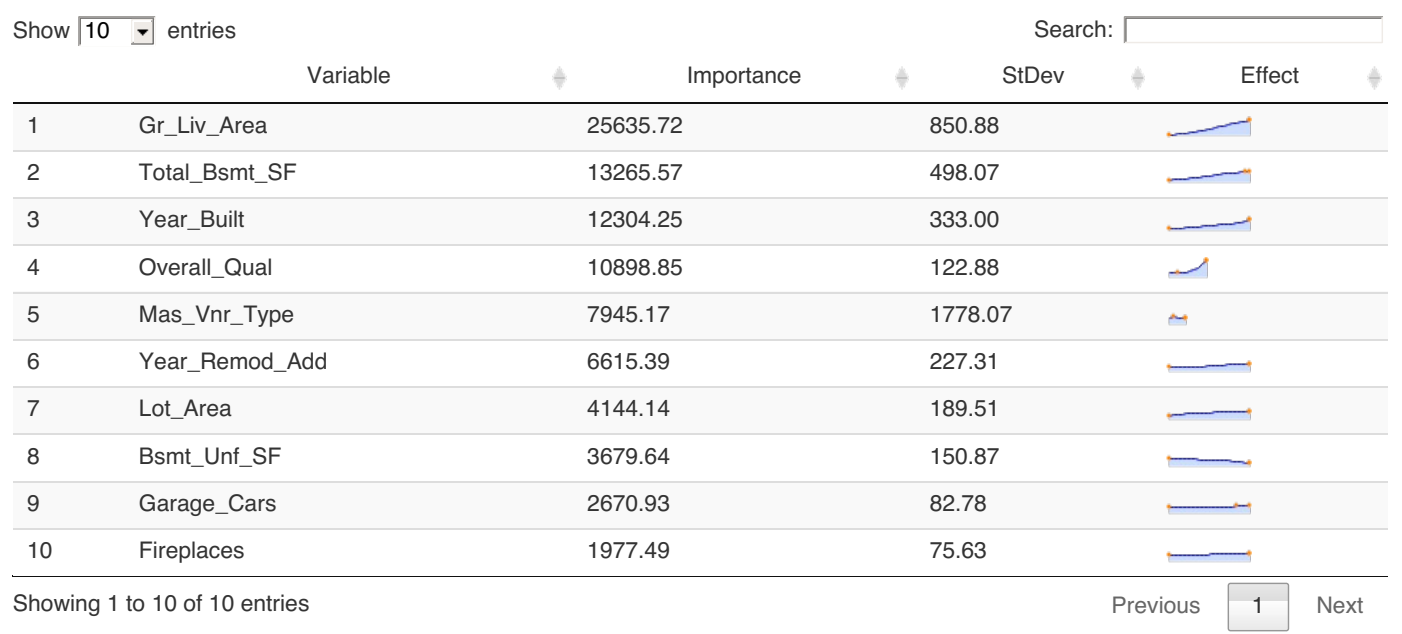

Figure 19: VIP with sparkline representation of feature effects for the top ten features from a Super Learner fit to the Ames housing data.

\# Shut down cluster

stopCluster $(\mathrm{cl})$

\section{Summary}

VIPs help to visualize the strength of the relationship between each feature and the predicted response, while accounting for all the other features in the model. We've discussed two types of VI: modelspecific and model-agnostic, as well as some of their strengths and weaknesses. In this paper, we showed how to construct VIPs for various types of "black box" models in R using the vip package. We also briefly discussed related approaches available in a number of other R packages. Suggestions to avoid high execution times were discussed and demonstrated via examples. This paper is based on vip version 0.2.2.9000. In terms of future development, vip can be expanded in a number of ways. For example, we plan to incorporate the option to compute group-based and conditional permutation scores. Although not discussed in this paper, vip also includes a promising statistic (similar to the variance-based VI scores previously discussed) for measuring the relative strength of interaction between features. Although VIPs can help understand which features are driving the model's predictions, ML practitioners should be cognizant of the fact that none of the methods discussed in this paper are uniformly best across all situations; they require an accurate model that has been properly tuned, and should be checked for consistency with human domain knowledge.

\section{Acknowledgments}

The authors would like to thank the anonymous reviewers and the Editor for their helpful comments and suggestions. We would also like to thank the members of the $84.51^{\circ}$ Interpretable Machine Learning Special Interest Group for their thoughtful discussions on the topics discussed herein.

\section{Bibliography}

D. Apley. ALEPlot: Accumulated Local Effects (ALE) Plots and Partial Dependence (PD) Plots, 2018. URL https://CRAN.R-project. org/package=ALEPlot. R package version 1.1. [p360] 
D. W. Apley and J. Zhu. Visualizing the effects of predictor variables in black box supervised learning models, 2016. [p359]

D. Bau, B. Zhou, A. Khosla, A. Oliva, and A. Torralba. Network dissection: Quantifying interpretability of deep visual representations. In The IEEE Conference on Computer Vision and Pattern Recognition (CVPR), July 2017. [p343]

A. Bibal and B. Frénay. Interpretability of machine learning models and representations: an introduction. In ESANN, 2016. [p343]

P. Biecek. DALEX: Descriptive mAchine Learning EXplanations, 2019. URL https: //CRAN. R-project. org/package=DALEX. R package version 0.4.9. [p343]

P. Biecek, H. Baniecki, and A. Izdebski. ingredients: Effects and Importances of Model Ingredients, 2019a. URL https: //CRAN. R-project. org/package=ingredients. R package version 0.5.0. [p343]

P. Biecek, A. Gosiewska, H. Baniecki, and A. Izdebski. iBreakDown: Model Agnostic Instance Level Variable Attributions, 2019b. URL https: //CRAN. R-project.org/package=iBreakDown. R package version 0.9.9. [p358]

B. Bischl, M. Lang, L. Kotthoff, J. Schiffner, J. Richter, Z. Jones, G. Casalicchio, M. Gallo, and P. Schratz. mlr: Machine Learning in R, 2020. URL https://CRAN. R-project.org/package=mlr. R package version 2.17.0. [p344]

L. Breiman. Bagging predictors. Machine Learning, 8(2):209-218, 1996. URL https://doi .org/10. 1023/A:1018054314350. [p344]

L. Breiman. Random forests. Machine Learning, 45(1):5-32, 2001. URL https: // doi . org/10. 1023/A: 1010933404324. [p354]

L. Breiman, J. Friedman, and R. A. O. Charles J. Stone. Classification and Regression Trees. The Wadsworth and Brooks-Cole statistics-probability series. Taylor \& Francis, 1984. ISBN 9780412048418. [p345]

R. Caruana, Y. Lou, J. Gehrke, P. Koch, M. Sturm, and N. Elhadad. Intelligible models for healthcare: Predicting pneumonia risk and hospital 30-day readmission. In Proceedings of the 21th ACM SIGKDD International Conference on Knowledge Discovery and Data Mining, KDD '15, pages 1721-1730, New York, NY, USA, 2015. Association for Computing Machinery. ISBN 9781450336642. URL https: //doi.org/10.1145/2783258.2788613. [p343]

E. Celik. vita: Variable Importance Testing Approaches, 2015. URL https://CRAN.R-project.org/ package=vita. R package version 1.0.0. [p344]

W. Chang. R6: Encapsulated Classes with Reference Semantics, 2019. URL https: //CRAN. R-project. org/ package=R6. R package version 2.4.1. [p343]

T. Chen, T. He, M. Benesty, V. Khotilovich, Y. Tang, H. Cho, K. Chen, R. Mitchell, I. Cano, T. Zhou, M. Li, J. Xie, M. Lin, Y. Geng, and Y. Li. xgboost: Extreme Gradient Boosting, 2019. URL https: //CRAN. Rproject. org/package=xgboost. R package version 0.90.0.2. [p359]

W. S. Cleveland. Robust locally weighted regression and smoothing scatterplots. Journal of the American Statistical Association, 74(368):829-836, 1979. doi: https://doi.org/10.1080/01621459.1979.10481038. [p344]

D. D. Cock. Ames, iowa: Alternative to the boston housing data as an end of semester regression project. Journal of Statistics Education, 19(3):1-15, 2011. URL https: //doi . org/10. 1080/10691898. 2011.11889627. [p360]

M. Corporation and S. Weston. doParallel: Foreach Parallel Adaptor for the 'parallel' Package, 2019. URL https://CRAN.R-project.org/package=doParallel. R package version 1.0.15. [p361]

G. Csárdi and M. Salmon. pkgsearch: Search and Query CRAN R Packages, 2019. URL https: //CRAN. Rproject. org/package=pkgsearch. R package version 3.0.2. [p344]

F. Doshi-Velez and B. Kim. Towards a rigorous science of interpretable machine learning, 2017. [p343]

M. Dowle and A. Srinivasan. data.table: Extension of 'data.frame', 2019. URL https: //CRAN. R-project. org/package=data. table. R package version 1.12.8. [p360]

A. Fisher, C. Rudin, and F. Dominici. Model class reliance: Variable importance measures for any machine learning model class, from the "rashomon" perspective. arXiv preprint arXiv:1801.01489, 2018. [p354] 
J. Friedman, T. Hastie, R. Tibshirani, B. Narasimhan, and N. Simon. glmnet: Lasso and Elastic-Net Regularized Generalized Linear Models, 2019. URL https://CRAN. R-project.org/package=glmnet. R package version 3.0-2. [p361]

J. H. Friedman. Multivariate adaptive regression splines. The Annals of Statistics, 19(1):1-67, 1991. URL https://doi.org/10.1214/aos/1176347963. [p344,349]

J. H. Friedman. Greedy function approximation: A gradient boosting machine. The Annals of Statistics, 29(5):1189-1232, 2001. URL https://doi .org/10.1214/aos/1013203451. [p351]

D. G. Garson. Interpreting neural-network connection weights. Artificial Intelligence Expert, 6(4):46-51, 1991. [p350]

T. Gedeon. Data mining of inputs: Analysing magnitude and functional measures. International Journal of Neural Systems, 24(2):123-140, 1997. URL https: //doi .org/10.1007/s10994-006-6226-1. [p350]

A. Goh. Back-propagation neural networks for modeling complex systems. Artificial Intelligence in Engineering, 9(3):143-151, 1995. URL https://dx. doi .org/10.1016/0954-1810(94)00011-S. [p350]

A. Goldstein, A. Kapelner, J. Bleich, and E. Pitkin. Peeking inside the black box: Visualizing statistical learning with plots of individual conditional expectation. Journal of Computational and Graphical Statistics, 24(1):44-65, 2015. URL https ://doi .org/10.1080/10618600.2014.907095. [p351, 352]

B. Greenwell. fastshap: Fast Approximate Shapley Values, 2019. URL https://github.com/bgreenwell/ fastshap. R package version 0.0.3.9000. [p358]

B. Greenwell, B. Boehmke, and B. Gray. vip: Variable Importance Plots, 2019. URL https://gi thub. com/ koalaverse/vip/. R package version 0.2.1. [p344]

B. M. Greenwell. pdp: An r package for constructing partial dependence plots. The R Journal, 9(1): 421-436, 2017. URL https://journal.r-project.org/archive/2017/RJ-2017-016/index. html. [p351]

B. M. Greenwell, B. C. Boehmke, and A. J. McCarthy. A simple and effective model-based variable importance measure. arXiv preprint arXiv:1805.04755, 2018. [p344, 351]

T. Hastie, R. Tibshirani, and J. Friedman. The Elements of Statistical Learning: Data Mining, Inference, and Prediction, Second Edition. Springer Series in Statistics. Springer-Verlag, 2009. [p345]

G. Hooker. Generalized functional anova diagnostics for high-dimensional functions of dependent variables. Journal of Computational and Graphical Statistics, 16(3):709-732, 2007. URL https: //doi. org/10.1198/106186007X237892. [p360]

G. Hooker and L. Mentch. Please stop permuting features: An explanation and alternatives, 2019. [p359]

T. Hothorn and A. Zeileis. partykit: A Toolkit for Recursive Partytioning, 2019. URL https://CRAN. Rproject. org/package=partykit. R package version 1.2-5. [p345]

T. Hothorn, K. Hornik, C. Strobl, and A. Zeileis. party: A Laboratory for Recursive Partytioning, 2019. URL https://CRAN. R-project.org/package=party. R package version 1.3-3. [p344]

D. Janzing, L. Minorics, and P. Blöbaum. Feature relevance quantification in explainable ai: A causal problem, 2019. [p359]

Z. Jones. mmpf: Monte-Carlo Methods for Prediction Functions, 2018. URL https://CRAN. R-project. org/package=mmpf. R package version 0.0.5. [p344]

A. Karatzoglou, A. Smola, and K. Hornik. kernlab: Kernel-Based Machine Learning Lab, 2019. URL https: //CRAN.R-project.org/package=kernlab. R package version 0.9-29. [p361]

A. Kozak and P. Biecek. vivo: Local Variable Importance via Oscillations of Ceteris Paribus Profiles, 2019. URL https://CRAN. R-project.org/package=vivo. R package version 0.1.1. [p351]

M. Kuhn. AmesHousing: The Ames Iowa Housing Data, 2017. URL https://CRAN. R-project.org/ package=AmesHousing. R package version 0.0.3. [p360]

M. Kuhn. caret: Classification and Regression Training, 2020. URL https://CRAN.R-project.org/ package=caret. $\mathrm{R}$ package version 6.0-85. [p344] 
M. Kuhn and K. Johnson. Applied Predictive Modeling. SpringerLink : Bücher. Springer New York, 2013. ISBN 9781461468493. [p344]

M. Lang, B. Bischl, J. Richter, P. Schratz, and M. Binder. mlr3: Machine Learning in R - Next Generation, 2019. URL https: //CRAN.R-project.org/package=mlr3. R package version 0.1.6. [p360]

M. Loecher. rfVarImpOOB: Unbiased Variable Importance for Random Forests, 2019. URL https: //CRAN. Rproject. org/package=rfVarImp00B. R package version 1.0. [p344]

S. M. Lundberg and S.-I. Lee. A unified approach to interpreting model predictions. In $A d-$ vances in Neural Information Processing Systems 30, pages 4765-4774. Curran Associates, Inc., 2017. URL http://papers.nips.cc/paper/7062-a-unified-approach-to-interpreting-modelpredictions.pdf. [p358]

S. M. Lundberg, G. Erion, H. Chen, A. DeGrave, J. M. Prutkin, B. Nair, R. Katz, J. Himmelfarb, N. Bansal, and S.-I. Lee. Explainable ai for trees: From local explanations to global understanding. arXiv preprint arXiv:1905.04610, 2019. [p358]

O. Mersmann. microbenchmark: Accurate Timing Functions, 2019. URL https: //CRAN. R-project. org/ package=microbenchmark. $\mathrm{R}$ package version 1.4-7. [p357]

S. Milborrow. earth: Multivariate Adaptive Regression Splines, 2019. URL https: //CRAN. R-project.org/ package=earth. R package version 5.1.2. [p349]

C. Molnar. iml: Interpretable Machine Learning, 2019a. URL https: //CRAN . R-project . org/package=iml. $\mathrm{R}$ package version 0.9.0. [p343]

C. Molnar. Interpretable Machine Learning. 2019b. https://christophm. github.io/interpretable$\mathrm{ml-book/.} \mathrm{[p343,354,356]}$

G. Montavon, W. Samek, and K.-R. Müller. Methods for interpreting and understanding deep neural networks. Digital Signal Processing, 73:1-15, 2018. URL https://doi .org/10.1016/j.dsp. 2017.10. 011. [p343]

K. Müller and H. Wickham. tibble: Simple Data Frames, 2019. URL https://CRAN. R-project.org/ package=tibble. R package version 2.1.3. [p344]

J. D. Olden, M. K. Joy, and R. G. Death. An accurate comparison of methods for quantifying variable importance in artificial neural networks using simulated data. Ecological Modelling, 178(3):389-397, 2004. URL https://dx.doi.org/10.1016/j.ecolmodel.2004.03.013. [p350]

A. Paluszynska, P. Biecek, and Y. Jiang. randomForestExplainer: Explaining and Visualizing Random Forests in Terms of Variable Importance, 2019. URL https://CRAN.R-project.org/package= randomForestExplainer. R package version 0.10.0. [p344]

T. Parr and J. D. Wilson. Technical report: A stratification approach to partial dependence for codependent variables, 2019. [p359]

E. Polley, E. LeDell, C. Kennedy, and M. van der Laan. SuperLearner: Super Learner Prediction, 2019. URL https: //CRAN. R-project.org/package=SuperLearner. R package version 2.0-26. [p361]

B. Poulin, R. Eisner, D. Szafron, P. Lu, R. Greiner, D. S. Wishart, A. Fyshe, B. Pearcy, C. MacDonell, and J. Anvik. Visual explanation of evidence in additive classifiers. In Proceedings of the 18th Conference on Innovative Applications of Artificial Intelligence - Volume 2, IAAI'06, pages 1822-1829. AAAI Press, 2006. [p343]

P. Probst. measures: Performance Measures for Statistical Learning, 2018. URL https: //CRAN. R-project. org/package=measures. R package version 0.2. [p344]

P. Probst. varImp: RF Variable Importance for Arbitrary Measures, 2019. URL https: //CRAN. R-project. org/package=varImp. R package version 0.3. [p344]

Revolution Analytics and S. Weston. foreach: Provides Foreach Looping Construct. [p343]

B. Ripley. nnet: Feed-Forward Neural Networks and Multinomial Log-Linear Models, 2016. URL https: //CRAN.R-project.org/package=nnet. R package version 7.3-12. [p350]

C. A. Scholbeck, C. Molnar, C. Heumann, B. Bischl, and G. Casalicchio. Sampling, intervention, prediction, aggregation: A generalized framework for model agnostic interpretations. CoRR, abs/1904.03959, 2019. URL http://arxiv.org/abs/1904.03959. [p351] 
C. Strobl, A.-L. Boulesteix, A. Zeileis, and T. Hothorn. Bias in random forest variable importance measures: Illustrations, sources and a solution. BMC Bioinformatics, 8(25), 2007. URL http: //www. biomedcentral.com/1471-2105/8/25. [p345]

C. Strobl, A.-L. Boulesteix, T. Kneib, T. Augustin, and A. Zeileis. Conditional variable importance for random forests. BMC Bioinformatics, 9(1):307, 2008. URL https://doi .org/10.1186/1471-2105-9307. [p344, 345, 359]

Q. Sun. tree.interpreter: Random Forest Prediction Decomposition and Feature Importance Measure, 2019. URL https://CRAN. R-project.org/package=tree. interpreter. R package version 0.1.0. [p344]

M. van der Laan. Statistical inference for variable importance. The International Journal of Biostatistics, 2 (1), 2006. URL https://doi.org/10.2202/1557-4679.1008. [p343]

H. Wickham. plyr: Tools for Splitting, Applying and Combining Data, 2019. URL https://CRAN.Rproject. org/package=plyr. R package version 1.8.5. [p361]

H. Wickham, W. Chang, L. Henry, T. L. Pedersen, K. Takahashi, C. Wilke, K. Woo, and H. Yutani. ggplot2: Create Elegant Data Visualisations Using the Grammar of Graphics, 2019. URL https: //CRAN. Rproject.org/package=ggplot2. R package version 3.2.1. [p344]

M. N. Wright, S. Wager, and P. Probst. ranger: A Fast Implementation of Random Forests, 2020. URL https://CRAN.R-project.org/package=ranger. R package version 0.12.1. [p344]

Y. Xie, J. Cheng, and X. Tan. DT: A Wrapper of the JavaScript Library 'DataTables', 2019. URL https: //CRAN.R-project.org/package=DT. R package version 0.11. [p360]

A. Zien, N. Kraemer, S. Sonnenburg, and G. Raetsch. The feature importance ranking measure, 2009. [p351]

E. Štrumbelj and I. Kononenko. Explaining prediction models and individual predictions with feature contributions. Knowledge and Information Systems, 31(3):647-665, 2014. URL https: //doi . org/10. 1007/s10115-013-0679-x. [p358]

\author{
Brandon M. Greenwell \\ University of Cincinnati \\ 2925 Campus Green Dr \\ Cincinnati, $\mathrm{OH} 45221$ \\ United States of America \\ ORCiD-0000-0002-8120-0084 \\ greenwell. brandon@gmail.com \\ Bradley C. Boehmke \\ University of Cincinnati \\ 2925 Campus Green Dr \\ Cincinnati, $\mathrm{OH} 45221$ \\ United States of America \\ ORCiD-0000-0002-3611-8516 \\ bradleyboehmke@gmail.com
}

